\title{
HOLOMORPHIC FUNCTIONS WITH VALUES \\ IN LOCALLY CONVEX SPACES AND APPLICATIONS TO INTEGRAL FORMULAS
}

\author{
BY
LUTZ BUNGART
}

1. Introduction. Holomorphic functions of one variable with values in a locally convex (Hausdorff topological vector) space (over the field $\mathbf{C}$ of complex numbers) have been studied before by A. Grothendieck [11]. However, A. Grothendieck is mainly concerned with the characterisation of the (topological) dual space of the space of holomorphic functions on an open (resp. compact) subset of the Riemann sphere having values in a locally convex space; and so are subsequent authors who generalize his ideas to Riemann surfaces and multicircular domains in several complex variables.

The purpose of our exposition is entirely different. We are concerned with properties of holomorphic functions and spaces of holomorphic functions with values in locally convex spaces which are defined on analytic spaces. We want to extend the basic results of $\mathrm{H}$. Cartan [5] to this more general class of functions and give some applications. Some of these problems have already been considered and solved in the existing literature for the very special case of families of holomorphic functions depending continuously on a parameter $t \in[0,1]$. Such families of holomorphic functions can be considered as holomorphic functions with values in the Banach space $\mathscr{C}([0,1])$ of continuous functions on the unit interval $[0,1]$.

We have to use some results from the theory of locally convex spaces not all of which are easily accessible. We recall some of the proofs (Proposition 3.1 and Lemma 5.1) for convenience.

In the first part of this thesis Theorems A and B of H. Cartan are generalized to analytic sheaves which are coherent over the sheaf of germs of holomorphic functions (on a Stein space $(X, \mathcal{O})$ ) with values in some Frechet space $E$. These coherent analytic sheaves are of the form $\mathscr{S} \varepsilon E$, where $\mathscr{S}$ is a coherent analytic sheaf on $X$ and $\mathscr{S} \varepsilon E$ is the sheaf defined by the presheaf $\{H(U, \mathscr{S}) \varepsilon E, U \subset X$ open $\}$. The $\varepsilon$-product used in the definition is the one defined by L. Schwartz; it is similar to the tensor product in algebra. For $E, F$ locally convex spaces, $F \otimes E$ is contained in $F \varepsilon E$. We usually assume that one of the spaces, say $F$, has a certain (nuclearity) property $(\mathscr{N})$. This is the case for instance if $F$ is taken

Received by the editors November $1,1962$. 
from the class of spaces $H(X, \mathscr{S})$ of cross sections of coherent analytic sheaves $\mathscr{S}$ over analytic spaces $(X, \mathcal{O})$. If we restrict $F$ to this class and $E$ to the class of Frechet spaces, then the $\varepsilon$-product is an exact functor. It is this fact which allows us to prove Theorems A and B. Some applications similar to those in the scalar case are given. The more general situation in which $E$ is only required to be a quasi-complete locally convex space is also studied.

The second part considers some applications to integral formulas. If $\left(Y, \mathcal{O}^{Y}\right)$ is a (closed) subvariety of the Stein space $(X, \mathcal{O})$, then the restriction map $H(X, \mathcal{O}) \rightarrow H\left(Y, \mathcal{O}^{Y}\right)$ has a continuous linear right inverse $B\left(Y, \mathcal{O}^{Y}\right) \rightarrow H(X, \mathcal{O})$, where $B\left(Y, \mathcal{O}^{Y}\right)$ is the Banach space of bounded functions in $H\left(Y, \mathbb{C}^{Y}\right)$. A choice of this map $f \rightarrow F$ can be represented by an integral formula $F(z)=\int_{Y} f d \eta_{Z}$, where $\eta$ is a holomorphic mapping from $X$ into the space of bounded measures on $Y$. By similar methods a Cauchy integral formula for a bounded subdomain $U$ of an analytic space is obtained; i.e., there is a holomorphic mapping $\eta$ from $U$ into the space of measures on the distinguished boundary $\Gamma$ of $U$ such that $f(z)=\int_{\Gamma} f d \eta_{z}, z \in U$, for a function $f$ holomorphic on the closure $\bar{U}$ of $U$. If $U$ is a bounded domain in $\mathbf{C}^{n}$ with $C^{\infty}$ boundary surface, then $\eta$ can be chosen to have values in the space of $C^{\infty}(2 n-1)$-forms on $\partial U$.

The last part of the author's thesis, which is concerned with boundary integral formulas for bounded domains in $\mathbf{C}^{n}$, will be published elsewhere since the methods used are independent of the technics developed in this paper.

Finally let us note that Theorem B and the immediate consequences have been obtained independently by E. Bishop (unpublished) using different methods. (Added in proof: The paper by E. Bishop Analytic functions with values in Frechet spaces has meanwhile appeared in Pacific J. Math. 12 (1962), 1177-1192.)

I would like to thank my supervisor, Professor H. Rossi, for his interest in my research, for his constant encouragement, and for the many helpful suggestions.

2. Notation. The notation and teminology from the theory of topological vector spaces is that of $\mathrm{N}$. Bourbaki [2]. All vector spaces will be vector spaces over the field $\mathbf{C}$ of complex numbers. We denote by $E^{\prime}$ the dual space of a locally convex space $E$, or space of continuous linear functionals in $E ; E_{s}\left(E_{s}^{\prime}\right)$ denotes the space $E\left(E^{\prime}\right)$ endowed with the topology of pointwise convergence on $E^{\prime}$ of $(E)$, the socalled weak topology for $E\left(E^{\prime}\right)$. For a set $B$ in $E, B^{0}$ is the polar of $B, B^{0}=\left\{y \in E^{\prime},|\langle x, y\rangle| \leqq 1\right.$ for $\left.x \in B\right\}$. The polar $B^{0} \subset E$ of a set $B \subset E^{\prime}$ is similarly defined.

The terminology in the theory of analytic spaces is the one employed in [14]. In particular, an analytic space is always supposed to be $\sigma$-compact. For the space of (continuous) cross sections of a sheaf $\mathscr{S}$ over a topological space $X$ we use the notation $H(X, \mathscr{S})$.

3. The $\varepsilon$-product of locally convex spaces. In the following let $E, F$ be locally convex (Hausdorff topological vector) spaces. We denote by $E_{c}^{\prime}$ the dual space $E^{\prime}$ 
of $E$ endowed with the topology of uniform convergence on compact discs (or convex circular sets) in $E . \mathscr{L}\left(E_{c}^{\prime}, F\right)$ is the vector space of continuous linear mappings $E_{c}^{\prime} \rightarrow F . \mathscr{L}_{\varepsilon}\left(E_{c}^{\prime}, F\right)$ denotes this space endowed with the topology of uniform convergence on the equicontinuous sets of linear functionals in $E^{\prime}$.

We are going to recall now some of the theorems in [16] for the convenience of the reader. We have [16, Corollary 2 to Proposition 4]

$$
\mathscr{L}_{\varepsilon}\left(E_{c}^{\prime}, F\right)=\mathscr{L}_{\varepsilon}\left(F_{c}^{\prime}, E\right),
$$

the isomorphism being given by $f \rightarrow f^{\prime}$, where $f^{\prime}$ denotes the transpose of $f$.

Let us recall the proof. Since $\left(E_{c}^{\prime}\right)^{\prime}=E$ by Mackey's Theorem [2], the transpose $f^{\prime}$ of $f \in \mathscr{L}_{\varepsilon}\left(E_{c}^{\prime}, F\right)$ is a linear map $F_{c}^{\prime} \rightarrow E$. Let us show that it is continuous. Choose a closed convex circular neighborhood $V$ of 0 in $E$.

$$
V^{0}\left(=\left\{y \in E^{\prime}:|\langle x, y\rangle| \leqq 1 \text { for } x \in V\right\}\right)
$$

is an equicontinuous set of linear functionals in $E^{\prime}$, compact for the weak topology, hence compact in $E_{c}^{\prime}$ which induces in $V^{0}$ the same topology as $E_{s}^{\prime}$. Now $f\left(V^{0}\right)=K$ is a compact disc in $F . K^{0}$ is a neighborhood of 0 in $F_{c}^{\prime}$ and $f^{\prime}\left(K^{0}\right) \subset V$. Thus $f^{\prime} \in \mathscr{L}_{\varepsilon}\left(F_{c}^{\prime}, E\right)$. By symmetry, the map $f \rightarrow f^{\prime}$ defines an isomorphism. Let us show that this isomorphism is continuous. Suppose $f$ tends to 0 in $\mathscr{L}_{\varepsilon}\left(E_{c}^{\prime}, F\right)$. Let $V^{0}$ be an equicontinuous set of linear functionals in $F^{\prime}$ ( $V$ a closed convex circular neighborhood of 0 in $F$ ), and $W$ a closed convex circular neighborhood of 0 in $E$. By assumption, $f$ maps $W^{0}$ finally into $V$, hence $f^{\prime}$ maps $V^{0}$ finally into $W$, i.e., $f^{\prime}$ converges to 0 in $\mathscr{L}_{\varepsilon}\left(F_{c}^{\prime}, E\right)$. Thus (3.1) follows by symmetry.

L. Schwartz [16] defines $E \varepsilon F$ in a symmetric way (which allows a simple proof of the associativity of this product) and then proves [16, Corollary 2 to Proposition 4]

$$
E \varepsilon F=\mathscr{L}_{\varepsilon}\left(E_{c}^{\prime}, F\right)=\mathscr{L}_{\varepsilon}\left(F_{c}^{\prime}, E\right),
$$

the last isomorphism being given by $f \rightarrow f^{\prime}$, where $f^{\prime}$ denotes the transpose of $f$. For our purposes, however, it suffices to take (3.2) as definition of the $\varepsilon$-product of two locally convex spaces $E$ and $F$.

Now suppose $F$ is a subspace of $E$. Then $F_{c}^{\prime}=E_{c}^{\prime} / F^{0}$; this can be seen as follows. Mackey's Theorem [2, Chapter 4, §2, No. 3] implies that the dual of $F_{c}^{\prime}$ as well as that of $E_{c}^{\prime} / F^{0}$ is $F$. The equicontinuous sets in $F$ as a dual of $F_{c}^{\prime}$ are those contained in compact discs in $F$, and as a dual of $E_{c}^{\prime} / F^{0}$ they are those contained in compact discs in $E$, i.e., the same. Thus $E_{c}^{\prime} / F^{0}=F_{c}^{\prime}$.

If $E_{1}, F_{1}$ are subspaces of $E$ resp. $F$, then $E_{1} \varepsilon F_{1}$ is a subspace of $E \varepsilon F$ as follows easily from the definition since the equicontinuous sets in $E_{1}^{\prime}=E^{\prime} / E_{1}^{0}$ are exactly the images of the equicontinuous sets in $E^{\prime}$.

Recall that if $E$ and $F$ are complete then so is $E \varepsilon F$. For if $\mathscr{U}$ is a Cauchy system in $\mathscr{L}_{\varepsilon}\left(E_{c}^{\prime}, F\right)$, then $\mathscr{U}$ converges uniformly on equicontinuous sets in $E^{\prime}$ to a linear 
map $f: E_{c}^{\prime} \rightarrow F$ (completeness of $F$ ). $f$ is continuous on equicontinuous sets in $E_{c}^{\prime}$. Let $\mathscr{U}^{\prime}$ be the image of $\mathscr{U}$ in $\mathscr{L}_{\varepsilon}\left(F_{c}^{\prime}, E\right)$ under the canonical isomorphism. $\mathscr{U}^{\prime}$ converges uniformly on equicontinuous sets in $F^{\prime}$ to a linear map $f^{\prime}: F_{c}^{\prime} \rightarrow E$. Considered as systems of bilinear functionals on $E^{\prime} \times F^{\prime}, \mathscr{U}=\mathscr{U}^{\prime}$ converges pointwise, hence $f=f^{\prime}$ as bilinear functionals on $E^{\prime} \times F^{\prime}$, i.e., $f^{\prime}$ is the transposed of $f$. It suffices now to prove continuity of $f^{\prime}$. Let $V$ be a closed convex circular neighborhood of 0 in $E$. $V^{0}$ is equicontinuous and compact in $E_{c}^{\prime}$. Since $f$ is continuous on $V^{0}, f\left(V^{0}\right)=K$ is a compact disc, and $f^{\prime}\left(K^{0}\right) \subset V$.

Thus if $E$ and $F$ are Frechet spaces, then so is $E \varepsilon F$; for $E \varepsilon F$ is complete and has a countable base of neighborhoods of 0 since $E^{\prime}$ has a countable base of equicontinuous sets $V^{0}$ ( $V$ in a countable base of neighborhoods of 0 in $E$ ).

A locally convex space $E$ is quasi-complete if the closed bounded sets in $E$ are complete. For instance, if $E$ is a Frechet space then $E_{s}^{\prime}$ is quasi-complete since the bounded sets in $E_{s}^{\prime}$ are equicontinuous and hence relatively compact in the weak topology. One important feature of quasi-complete locally convex spaces is that the closed convex circular hull of a compact set is compact; for it is easily shown to be totally bounded, and since closed bounded sets are complete, it must be compact also. In particular, $E_{c}^{\prime}$ has the topology of uniform convergence on all compact sets in $E$ if $E$ is quasi-complete.

We have for any quasi-complete space $E$ [10, Chapter I, pp. 89-90] :

PROPOSITION 3.1. Suppose $X$ is a locally compact $\sigma$-compact Hausdorff space. Let $\mathscr{C}(X, E)$ be the space of continuous $E$-valued functions on $X$ with the topology of uniform convergence on compact sets. There is a canonical isomorphism

$$
\mathscr{C}(X, E)=\mathscr{C}(X) \varepsilon E \quad(\mathscr{C}(X)=\mathscr{C}(X, \mathbf{C})) .
$$

Proof. Note first that we have a canonical continuous map $j: X \rightarrow \mathscr{C}(X)_{c}^{\prime}$ mapping $x \in X$ onto the unit point mass at $x$ (it is continuous since it maps compact sets into equicontinuous sets for which $\mathscr{C}(X)_{c}^{\prime}$ and $\mathscr{C}(X)_{s}^{\prime}$ induce the same topology). We define

$$
i: \mathscr{L}_{\varepsilon}\left(\mathscr{C}(X)_{c}^{\prime}, E\right) \rightarrow \mathscr{C}(X, E)
$$

by $i(f)=f \circ j . i$ is injective; this follows in the case $E=\mathbf{C}$ from $\left[\mathscr{C}(X)_{c}^{\prime}\right]^{\prime}=\mathscr{C}(X)$. In general, if $i(f)=0$ then $0=\langle i(f), y\rangle=i(\langle f, y\rangle)$, i.e., $\langle f, y\rangle=0$ for all $y \in E^{\prime}$; hence $f=0 . i$ is also onto; for let $f \in \mathscr{C}(X, E)$, then $y \rightarrow\langle f, y\rangle$ defines a linear map $g: E_{c}^{\prime} \rightarrow \mathscr{C}(X) . g$ is continuous, for if $V=\{h \in \mathscr{C}(X):|h(x)| \leqq 1$ for $x \in K\}$, $K \subset X$ compact, then $f(K)$ is contained in a compact disc $K_{1}$ in $E$, and $g\left(K_{1}^{0}\right) \subset V$. Obviously, $i\left(g^{\prime}\right)=f\left(g^{\prime}\right.$ is the transpose of $\left.g\right)$. $i$ is also a homeomorphism; $f^{\prime}$ converges to 0 in $\mathscr{L}_{\varepsilon}\left(E_{c}^{\prime}, \mathscr{C}(X)\right)$ if and only if $\langle f, y\rangle$ converges to 0 in $\mathscr{C}(X)$ uniformly as $y$ stays in an equicontinuous set in $E^{\prime}$, i.e., if and only if $i(f)$ converges to 0 in $\mathscr{C}(X, E)$. 
4. Digression on holomorphic functions. Let $E$ be a locally convex space. We denote by $H\left(P^{n} ; E\right)$ the space of holomorphic $E$-valued functions (convergent power series with coefficients in $E$ ) on a polycylinder $P^{n}$ in $\mathbf{C}^{n}$, endowed with the topology of uniform convergence on compact sets. We write $H\left(P^{n}\right)$ instead of $H\left(P^{n} ; \mathbf{C}\right)$. We have

THEOREM 4.1. Let E be a quasi-complete locally convex space, $P^{n}$ a polycylinder in $\mathbf{C}^{n}$. The following are equivalent for an E-valued function $f$ on $P^{n}$ :

(1) $\langle f, y\rangle$ is holomorphic for every $y \in E^{\prime}$;

(2) $f \in H\left(P^{n} ; E\right)$;

(3) $f \in \mathscr{L}_{\varepsilon}\left(E_{c}^{\prime}, H\left(P^{n}\right)\right)=H\left(P^{n}\right) \varepsilon E$.

Proof. (1) implies (2): This is proven in [11]. A similar argument is carried out in the proof of Corollary 19.3.

(2) implies (3). We know from Proposition $3.1, \mathscr{C}\left(P^{n}, E\right)=\mathscr{L}_{\varepsilon}\left(E_{c} ; \mathscr{C}\left(P^{n}\right)\right)$. Clearly, to the subspace $H\left(P^{n} ; E\right)$ of $\mathscr{C}\left(P^{n}, E\right)$ corresponds the subspace $\mathscr{L}_{\varepsilon}\left(E_{c}^{\prime}, H\left(P^{n}\right)\right)$ of $\mathscr{L}_{\varepsilon}\left(E_{c}^{\prime}, \mathscr{C}\left(P^{\eta}\right)\right)$ under this isomorphism.

(3) implies (1) is trivial.

Corollary 4.2. Suppose $E$ is a quasi-complete locally convex space; then

$$
H\left(P^{n}\right) \varepsilon E=H\left(P^{n} ; E\right) \text {. }
$$

Proof. The isomorphism in the algebraic sense is assured by Theorem 4.1, and in the topological sense by Proposition 3.1.

A similar theorem for analytic spaces will be proved later (Theorem 16.1). Let us, however, make the following definition.

Definition 4.3. Let $(X, \mathcal{O})$ be an analytic space. We define $H(X, \mathcal{O}) \varepsilon E$ to be the space of holomorphic functions on $X$ with values in the quasi-complete locally convex space $E$, where we consider $H(X, \mathcal{O})$ with the topology induced by $\mathscr{C}(X)$. $\mathcal{O}^{E}$ denotes the sheaf of germs of such functions.

Note that $H(X, \mathcal{O}) \varepsilon E$ is a subspace of $\mathscr{C}(X) \varepsilon E=\mathscr{C}(X, E)$ (Proposition 3.1) under canonical identifications. $H(X, \mathcal{O})$ is a closed subspace of $\mathscr{C}(X)$; (for further details the reader is referred to $\S 7)$. Thus $H(X, \mathcal{O}) \varepsilon E$ is a Frechet space if $E$ is as we have noted in $\$ 3$.

5. Spaces of type $(\mathscr{N})$. We say that a locally convex space $E$ has the inverse limit topology of a system of Hilbert spaces if there are a family of Hilbert spaces $\left\{H_{\alpha}\right\}$ and continuous linear maps $u_{\alpha}: E \rightarrow H_{\alpha}$ such that the topology of $E$ is the weakest locally convex topology which makes all maps $u_{\alpha}$ continuous.

A locally convex space $E$ is said to be of type $(\mathscr{N})$ if $E_{c}^{\prime}$ has the inverse limit topology of a system of Hilbert spaces $\left\{H_{\alpha}\right\}$ with the property:

$(\mathscr{N})$ For each $\alpha$ there is an $\alpha_{1}$ and a map $u_{\alpha \alpha_{1}}: H_{\alpha_{1}} \rightarrow H_{\alpha}$ of the form

$$
u_{\alpha \alpha_{1}}=\sum_{i=1}^{\infty} \lambda_{i} e_{i} \otimes f_{i}
$$


where $\left\{e_{i}\right\} \subset \bar{H}_{\alpha_{1}}, \quad\left\{f_{i}\right\} \subset H_{\alpha}$ are bounded and $\Sigma\left|\lambda_{i}\right|<\infty$, such that $u_{\alpha}=u_{\alpha \alpha_{1}} \circ u_{\alpha_{1}}$ (here $\bar{H}_{\alpha_{1}}$ is the dual of $H_{\alpha_{1}}$ ).

By Mackey's Theorem [2, Chapter 4, §2, No. 3], the dual of $E_{c}^{\prime}$ coincides with $E$. Let $e_{i}^{0}$ be the image of $e_{i}$ via the transpose $\bar{H}_{\alpha_{1}} \rightarrow E$ of $u_{\alpha_{1}}$. Then $\left\{e_{i}^{0}\right\}$ is contained in a compact disc by the definition of the topology of $E_{c}^{\prime}$; hence we can write

$$
u_{\alpha}=\sum \lambda_{i} e_{i}^{\dot{v}} \otimes f_{i} .
$$

Such maps are called nuclear in $[10 ; 5]$. In the terminology of $[10 ; 15], E$ is of type $(\mathscr{N})$ if and only if $E_{c}^{\prime}$ is nuclear, which in the case where $E$ is a Frechet space is equivalent to saying that $E$ is nuclear.

We may assume that the sets $u_{\alpha}^{-1}\left(W_{\alpha}\right)$, where $W_{\alpha}$ is the unit ball in $H_{\alpha}$, form a base for the neighborhoods of 0 in $E_{c}^{\prime}$; we may replace the family $\left\{H_{\alpha}\right\}$ (respectively $\left\{u_{\alpha \alpha_{1}}\right\}$ ) by the family of finite direct sums of spaces $H_{\alpha}$ (respectively of maps $\left.u_{\alpha \alpha_{1}}\right)$. Also, we may assume that $u_{\alpha}\left(E_{c}^{\prime}\right)$ is dense in $H_{\alpha}$; if not, we replace $H_{\alpha}$ by $\overline{u_{\alpha}\left(E_{c}^{\prime}\right)}$. The maps $u_{\alpha \alpha_{1}}$ are then given by

$$
\sum \lambda_{i} p_{\alpha_{1}}^{*}\left(e_{i}\right) \otimes p_{\alpha}\left(f_{i}\right)
$$

where $p_{\alpha}$ is the orthogonal projection onto $\overline{u_{\alpha}\left(E_{c}^{\prime}\right)}$ and * denotes the adioint.

LemMa 5.1. Let $F$ be a closed subspace of a Frechet space $E$ of type $(\mathscr{N})$; then $F$ and $E / F$ are of type $(\mathscr{N})$.

Proof. Let $E_{c}^{\prime}$ have the inverse limit topology of the system of Hilbert spaces $\left\{H_{\alpha}\right\}$ with maps $u_{\alpha}: E_{c}^{\prime} \rightarrow H_{\alpha}$, and the family of maps $\left\{u_{\alpha \alpha_{1}}\right\}$ having property $(\mathscr{N})$. Let $H_{\alpha}^{1}=u_{\alpha}\left(F^{\perp}\right), H_{\alpha}^{2}=H_{\alpha} / H_{\alpha}^{1} \cong H_{\alpha}^{1 \perp} \subset H_{\alpha}$, and $u_{\alpha \alpha_{1}}^{i}: H_{\alpha_{1}}^{i} \rightarrow H_{\alpha}^{i}$ the maps derived from $u_{\alpha \alpha_{1}}$ in the obvious way. Then the $u_{\alpha \alpha_{1}}^{i}$ have again property $(\mathscr{N})$ :

$$
\begin{aligned}
& u_{\alpha \alpha_{1}}^{1}=\sum \lambda_{i} p_{\alpha_{1}}^{*}\left(e_{i}\right) \otimes p_{\alpha}\left(f_{i}\right), \\
& u_{\alpha \alpha_{1}}^{2}=\sum \lambda_{i}\left(1-p_{\alpha_{1}}^{*}\right)\left(e_{i}\right) \otimes\left(1-p_{\alpha}\right)\left(f_{i}\right),
\end{aligned}
$$

$p_{\alpha}$ being the orthogonal projection onto $H_{\alpha}^{1}$.

Let $u_{\alpha}^{1}: F^{\perp} \rightarrow H_{\alpha}^{1}$ and $u_{\alpha}^{2}: E^{\prime} / F^{\perp} \rightarrow H_{\alpha}^{2}$ be the maps derived from $u_{\alpha}: E^{\prime} \rightarrow H_{\alpha}$. Then $F_{c}^{\prime}$ has the inverse limit topology of the $\left\{H_{\alpha}^{2}\right\}$ and $(E / F)_{c}^{\prime}$ the inverse limit topology of the $\left\{H_{\alpha}^{1}\right\}$ if we can show that the topological isomorphisms $F_{c}^{\prime}=E_{c}^{\prime} / F^{\perp}$ and $(E / F)_{c}^{\prime}=F^{\perp} \subset E_{c}^{\prime}$ hold. $E$ is a Frechet space, so the second isomorphism holds since any compact set in $E / F$ is the image of a compact set in $E$. The first isomorphism holds for any locally convex space $E$ as we have recalled in $\S 3$.

CoROllary 5.2. Every closed subspace, quotient space, or closed subspace of a quotient space of $\left[H\left(P^{n}\right)\right]^{q}$ has the property $(\mathscr{N})$.

Proof. We have to show that $H\left(P^{n}\right)$ has the property $(\mathscr{N})$. Let $\alpha$ be a continuous function on $P^{n}$ such that $|\alpha|>0$. Define $\bar{H}_{\alpha}$ to be the subspace of $H\left(P^{n}\right)$ of functions $f$ satisfying 


$$
\int_{p^{n}}|\alpha f|^{2}<\infty
$$

$\bar{H}_{\alpha}$ is a Hilbert space with inner product

$$
(f, g)=\int_{p^{n}} \alpha f \cdot \bar{\alpha} \bar{f} .
$$

The unit ball $W_{\alpha}$ in $\bar{H}_{\alpha}$ is a relatively compact set in $H\left(P^{n}\right)$, and the set of all $\bar{W}_{\alpha}$ (closure in $H\left(P^{n}\right)$ ) is a base for the compact sets in $H\left(P^{n}\right)$, every compact set in $H\left(P^{n}\right)$ is contained in one of the $W_{\alpha}$. Hence, if $H_{\alpha}$ denotes the dual of $\bar{H}_{\alpha}$, and $u_{\alpha}: H\left(P^{\eta}\right)_{c}^{\prime} \rightarrow H_{\alpha}$ the transpose of the injection $\bar{H}_{\alpha} \rightarrow H\left(P^{n}\right)$, then $H\left(P^{n}\right)_{c}^{\prime}$ has the inverse limit topology of the system $\left\{H_{\alpha}\right\}$.

Now consider the linear functional $d_{k} \in H\left(P^{n}\right)_{c}^{\prime}, k=\left(k_{1}, \cdots, k_{n}\right)$ defined by $d_{k}(f)=f^{k}(0)$, where $f^{k}$ denotes the $k$ th derivative of $f$. By Cauchy's formula we have an estimate

$$
\left|d_{k}(f)\right| \leqq M r^{-(|k|+n)} M_{f, r}, \quad|k|=\sum k_{i}
$$

where $M_{f . r}$ is the supremum of $|f|$ on the polycylinder of radius $r<1$, and $M$ a constant independent of $f$ and $r$. If we restrict $f$ to $W_{\alpha}$, we can find a (multiple) sequence $r_{k}<1$ tending to 1 and a constant $K_{\alpha}$ such that

$$
r_{k}^{|k|} M_{f, r_{k}} \leqq K_{\alpha}
$$

Then $r_{k}^{2|k|} d_{k}$ is bounded in $H_{\alpha}$ and $r_{k}^{-|k|} z^{k}\left(z^{k}=z_{1}^{k_{1}} \cdots z_{n}^{k_{n}}\right)$ is bounded in $H\left(P^{n}\right)$, hence bounded in some $\bar{H}_{\beta}$. We may assume $\bar{H}_{\beta} \supset \bar{H}_{\alpha}$. Then the natural map $H_{\beta} \rightarrow H_{\alpha}$ can be written

$$
\sum \frac{1}{k !} r_{k}^{-|k|} z^{k} \otimes r_{k}^{|k|} d_{k}
$$

We can now prove the following theorem which is a special case of a Künneth Theorem proved by A. Grothendieck [16].

TheOREM 5.3. Suppose $E_{1}, E_{2}, E_{3}, F$ are Frechet spaces and

$$
0 \rightarrow E_{1} \rightarrow E_{2} \rightarrow E_{3} \rightarrow 0
$$

is exact. If either $E_{3}$ or $F$ has the property $(\mathscr{N})$, then

$$
0 \rightarrow E_{1} \varepsilon F \rightarrow E_{2} \varepsilon F \rightarrow E_{3} \varepsilon F \rightarrow 0
$$

is exact.

Proof. We have already remarked that $E_{1} \varepsilon F$ is a subspace of $E_{2} \varepsilon F$. Exactness in the middle of (5.1) is also immediately verified if we remember that $E_{2} \varepsilon F$ $=\mathscr{L}_{\varepsilon}\left(F_{c}^{\prime}, E_{2}\right)$.

So we have only to check that

$$
\mathscr{L}_{\varepsilon}\left(F_{c}^{\prime}, E_{2}\right) \rightarrow \mathscr{L}_{\varepsilon}\left(F_{c}^{\prime}, E_{3}\right)
$$


is onto. Let $U_{n}$ be a fundamental sequence of neighborhoods of 0 in $E_{3}$ and $f \in \mathscr{L}_{\varepsilon}\left(F_{c}^{\prime}, E_{3}\right)$. The polars $\left(f^{-1}\left(U_{n}\right)\right)^{0}=K_{n}=\{x \in F:|(x, y)| \leqq 1$ for $\left.y \in f^{-1}\left(U_{n}\right)\right\}$ are compact in $F$. Since $F$ is a Frechet space, we can find a sequence

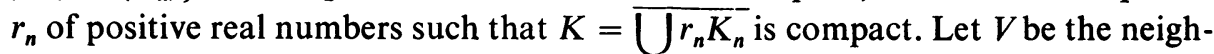
borhood in $F_{c}^{\prime}$ defined by $K$. Then $f: F^{\prime} \rightarrow E_{3}$ is continuous if we consider $F^{\prime}$ with the topology generated by $V$. Now assume $F$ is of type $(\mathscr{N})$, say $F_{c}^{\prime}$ has the inverse limit topology of the system $\left\{H_{\alpha}\right\}$ of Hilbert spaces, with maps $u_{\alpha}: F_{c}^{\prime} \rightarrow H_{\alpha}$. Suppose also that $u_{\alpha}\left(F_{c}^{\prime}\right)$ is dense in $H_{\alpha}$. Then there is an $\alpha$ such that $f: F_{c}^{\prime} \rightarrow E_{3}$ splits

$$
F_{c}^{\prime} \stackrel{u_{\alpha}}{\rightarrow} H_{\alpha} \stackrel{g}{\rightarrow} E_{3}
$$

Since

$$
u_{\alpha}=\sum \lambda_{i} f_{i} \otimes h_{i}, \quad \sum\left|\lambda_{i}\right|<\infty,
$$

$\left\{f_{i}\right\}$ bounded in $F,\left\{h_{i}\right\}$ bounded in $H_{\alpha}$, we can write

$$
f=\sum \lambda_{i} f_{i} \otimes g_{i}, \quad g_{i}=g\left(h_{i}\right),
$$

where $\left\{g_{i}\right\}$ is bounded in $E_{3}$ and the sum converges in $\mathscr{L}_{\varepsilon}\left(F_{c}^{\prime}, E_{3}\right)$. Let $g_{i}^{0}: L_{2}$ map onto $g_{i}$. We may assume that $\left\{g_{i}^{0}\right\}$ is bounded in $E_{2}$. Then

$$
f^{0}=\sum \lambda_{i} f_{i} \otimes g_{i}^{0}
$$

defines an element of $E_{2} \varepsilon F=\mathscr{L}_{\varepsilon}\left(F_{c}^{\prime}, E_{2}\right)$ which maps onto $f$.

In the construction of the series (5.2) we may exchange the roles of $F$ and $E_{3}$; but we have then to lift the $f_{i}$ instead of the $g_{i}$. This proves the theorem.

6. Frechet sheaves [14, Chapter 2], and topological sheaves. Let $X$ be a topological space satisfying the second axiom of countability. A Frechet sheaf, or a sheaf of type $(\mathscr{F})$, over $X$ is a sheaf $\mathscr{F}$ with the following properties. There is a collection of Frechet spaces $\{\mathscr{F}(U), U \in \mathscr{U}\}, \mathscr{U}$ a basis of open sets for $X$, such that $\left(\mathscr{F}_{1}\right) \mathscr{F}(U)=H(U, \mathscr{F})$ for $U \in \mathscr{U}$;

$\left(\mathscr{F}_{2}\right)$ if $U \subset V$ and $U, V \in \mathscr{U}$, then the restriction map

$$
r_{V U}: \mathscr{F}(V) \rightarrow \mathscr{F}(U)
$$

is continuous.

Here $H(U, \mathscr{F})$ denotes the space of (continuous) cross sections of $\mathscr{F}$ over $U$. One checks easily (using the open mapping theorem)

$\left(\mathscr{F}_{3}\right)$ if $V \in \mathscr{U}$, then $\mathscr{F}(V)$ has the inverse limit topology with respect to the mappings $r_{V U}: \mathscr{F}(V) \rightarrow \mathscr{F}(U), U \in \mathscr{V}$, where $\mathscr{V} \subset \mathscr{U}$ is any basis of open sets in $V$.

One can define topological sheaves in a similar way, requiring only that $\mathscr{F}(U)$ be a locally convex space, but adding $\left(\mathscr{F}_{3}\right)$ as an axiom.

The property $\left(\mathscr{F}_{3}\right)$ justifies the following definition of a topology for $H(V, \mathscr{F})$, where $V$ is any open subset of $X$ : 
$H(V, \mathscr{F})$ has the inverse limit topology with respect to the family of linear maps $r_{V U}: H(V, \mathscr{F}) \rightarrow H(U, \mathscr{F})=\mathscr{F}(U), U \in \mathscr{U}, U \subset V$.

If $\mathscr{F}$ is a Frechet sheaf, $H(V, \mathscr{F})$ becomes thus a Frechet space, as is easily verified.

Suppose $X$ is a locally compact Hausdorff space. The topological sheaf $\mathscr{F}$ on $X$ is said to be of type $(\mathscr{S})$ if the following holds:

$(\mathscr{S})$ Suppose $U, V \in \mathscr{U}, \bar{V} \subset U$, and $\bar{V}$ compact, then the restriction map $H(U, \mathscr{F}) \rightarrow H(V, \mathscr{F})$ is compact (i.e., the image of some neighborhood of 0 in $H(U, \mathscr{F})$ is totally bounded in $H(V, \mathscr{F}))$.

A simple compactness argument shows that $(\mathscr{S})$ holds for a sheaf of type $(\mathscr{S})$ also if we replace $\mathscr{U}$ by the system of all open subsets of $X$.

Proposition 6.1. Suppose $\mathscr{F}$ is a Frechet sheaf of type $(\mathscr{S})$ on the locally compact Hausdorff space $X$, and $X$ is second countable. Then for any open set $U \subset X, H(U, \mathscr{F})$ is of type $(\mathscr{M})($ Montel $)$, i.e., the closed bounded sets in $H(U, \mathscr{F})$ are compact.

Proof. We may assume that $U=X$. Let $K \subset H(X, \mathscr{F})$ be a closed bounded set, and $U_{n} \subset X$ a sequence of relatively compact sets such that $\bar{U}_{n} \subset U_{n+1}$, $\bigcup U_{n}=X$. We denote the restriction map $H(X, \mathscr{F}) \rightarrow H\left(U_{n}, \mathscr{F}\right)$ by $r_{n}$. Since $r_{n}$ is compact, $r_{n}(K)$ is relatively compact in $H\left(U_{n}, \mathscr{F}\right)$. If $\mathscr{U}$ is an ultra-filter in $K$, then $r_{n}(\mathscr{U})$ converges to a point $y_{n} \in \overline{r_{n}(K)}$. Clearly $y_{n+1}$ maps onto $y_{n}$ under the restriction map $H\left(U_{n+1}, \mathscr{F}\right) \rightarrow H\left(U_{n}, \mathscr{F}\right)$. Since $H(X, \mathscr{F})$ is the inverse limit of the spaces $H\left(U_{n}, \mathscr{F}\right)$, there is a $y \in H(X, \mathscr{F})$ with $y_{n}=r_{n}(y)$. By the definition of the topology, $\mathscr{U} \rightarrow y \in K$.

7. Coherent analytic sheaves. For example, let $G \neq \varnothing$ be an open subset of $\mathbf{C}^{n}$. We denote by $\mathcal{O}^{q}$ the sheaf of $q$-tuples of germs of holomorphic functions on $G$. $\mathcal{O}^{q}$ is a Frechet sheaf since it can be defined by the pre-sheaf

$$
\left\{H\left(P_{x}^{n}\right)^{q}, x \in G, P_{x}^{n} \subset G \text { a polycylinder with center } x\right\} .
$$

In fact, every coherent analytic subsheaf $\mathscr{S}$ of $\mathscr{O}^{q}$ is a Frechet sheaf in the induced structure, that is, if we give $H(U, \mathscr{S}), U \subset G$ open, the topology induced by $H\left(U, \mathcal{O}^{q}\right)$. This is a consequence of a theorem of $\mathrm{H}$. Cartan [5] on the closure of modules stating that if $\mathfrak{M}$ is a submodule of $\mathcal{O}_{x}^{q}, x \in U$, and $f_{k} \in H\left(U, \mathcal{O}^{q}\right)$ a sequence tending to $f \in H\left(U, \mathcal{O}^{q}\right)$ such that $\left(f_{k}\right)_{x} \in \mathfrak{M}$ for each $k$, then $f_{x} \in \mathfrak{M}$. Moreover, $\mathscr{S}$ is of type $(\mathscr{S})$ by the theorem of Montel.

Now let $\mathscr{S}$ be any coherent analytic sheaf on an analytic subvariety $X$ of a polycylinder $P^{n}$ in $\mathbf{C}^{n}$ and suppose there is an exact sequence

$$
0 \rightarrow \mathscr{K} \rightarrow \mathcal{O}_{n}^{q} \rightarrow \mathscr{S} \rightarrow 0
$$

where $\mathcal{O}_{n}$ is now the sheaf of germs of holomorphic functions on $P^{n}$, and $\mathscr{S}$ is 
defined to be trivial on $P^{n}-X$. For $U \subset P^{n}$ a polycylindric neighborhood of any point in $P^{n}$,

$$
0 \rightarrow H(U, \mathscr{K}) \rightarrow H\left(U, \mathcal{O}_{n}^{q}\right) \rightarrow H(U, \mathscr{S}) \rightarrow 0
$$

is exact by Theorem B $[5 ; 14]$. So we can take for $H(U \cap X, \mathscr{S})=H(U, \mathscr{S})$ the quotient topology $\left(H(U, \mathscr{K})\right.$ is a closed subspace of $H\left(U, \mathcal{O}_{n}^{q}\right)$ since $\mathscr{K}$ is a coherent analytic subsheaf of $\left.\mathcal{O}_{n}^{q}\right)$. Suppose

$$
0 \rightarrow \mathscr{K}_{0} \rightarrow \mathscr{O}_{n}^{p} \rightarrow \mathscr{S} \rightarrow 0
$$

is another exact sequence. Then there are homomorphisms

$$
h: \mathcal{O}_{n}^{q} \rightarrow \mathcal{O}_{n}^{p}, \quad h^{\prime}: \mathcal{O}_{n}^{p} \rightarrow \mathcal{O}_{n}^{q}
$$

which make the diagram

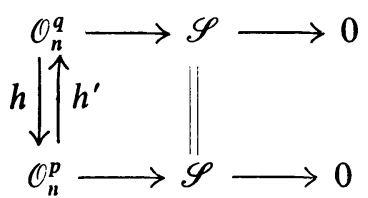

commutative. $h, h^{\prime}$ induce continuous maps

$$
h: H\left(U, \mathcal{O}_{n}^{q}\right) \rightarrow H\left(U, \mathcal{O}_{n}^{p}\right), h^{\prime}: H\left(U, \mathcal{O}_{n}^{p}\right) \rightarrow H\left(U, \mathcal{O}_{n}^{q}\right),
$$

which pass to the quotients giving continuous linear maps $H\left(U, \mathcal{O}_{n}^{q}\right) / H(U, \mathscr{K})$ $\rightarrow H\left(U, \mathcal{O}_{n}^{p}\right) / H\left(U, \mathscr{K}_{0}\right)$ and $H\left(U, \mathcal{O}_{n}^{p}\right) / H\left(U, \mathscr{K}_{0}\right) \rightarrow H\left(U, \mathcal{O}_{n}^{q}\right) / H(U, \mathscr{K})$; i.e., the topology for $H(U, \mathscr{S})$ is independent of the choice of (7.1). Axioms $\left(\mathscr{F}_{1}\right)$ and $\left(\mathscr{F}_{2}\right)$ are now easily verified. Also $\mathscr{S}$ is of type $(\mathscr{S})$ since $H(U, \mathscr{S})$ is a quotient of $H\left(U, \mathcal{O}_{n}^{q}\right)$ in the above, and $\mathcal{O}_{n}^{q}$ is of type $(\mathscr{S})$.

If $\mathscr{S}$ is a coherent analytic subsheaf of $\mathscr{O}^{q}$ on $X$, then $\mathscr{S}$ is also a subsheaf of the sheaf $\mathscr{C}^{q}$ of germs of $q$-tuples of continuous functions on $X . \mathscr{C}^{q}$ is a Frechet sheaf in a natural way (compact open topology for $H\left(U, \mathscr{C}^{q}\right), U \subset X$ open). Thus $\mathscr{S}$ has an induced structure from $\mathscr{C}^{q}$. H. Grauert and R. Remmert have proved in [8] that the natural continuous injection $H(U, \mathcal{O}) \rightarrow H(U, \mathscr{C})$ maps $H(U, \mathcal{O})$ onto a closed subspace of $H(U, \mathscr{C})$ and is thus a homeomorphism by the open mapping theorem (for an elementary proof see [4]). Hence $\mathscr{C}^{q}$ induces a Frechet sheaf structure in $\mathscr{S}$ which is identical to the one defined above.

Now let $(X, \mathcal{O})$ be any analytic space and $\mathscr{S}$ a coherent analytic sheaf on $X$. We can cover $X$ by open sets $X_{j}$ which can be embedded into polycylinders and such that there are exact sequences (7.1) for $\mathscr{S} \mid X_{j}$. Thus we can define for $\mathscr{S} \mid X_{j}$ the structure of a Frechet sheaf. These structures coincide on $X_{j} \cap X_{i}$ since for $U \subset X_{j} \cap X_{i}$ a Stein subdomain, $H(U, \mathscr{S})$ is a quotient of $H\left(U, \mathscr{O}^{q}\right)$ and the topology of $H\left(U, \mathscr{O}^{q}\right)$ is uniquely determined by that of $H\left(U, \mathscr{C}^{q}\right)$. Thus $\mathscr{S}$ is a Frechet sheaf of type $(\mathscr{S})$. 
8. Sheaves of type $(\mathscr{N})$. A topological sheaf $\mathscr{F}$ over the topological space $X$ is said to be of type $(\mathscr{N})$ if for a base of open sets $U \subset X, H(U, \mathscr{F})$ is of type $(\mathscr{N})$ (§5). We have by Corollary 5.2

Proposition 8.1. Let $(X, \mathcal{O})$ be an analytic space, $\mathscr{S}$ a coherent analytic sheaf on $X$. Then $\mathscr{S}$ is of type $(\mathscr{N})$.

We need the following fact:

Proposition 8.2. Suppose the sheaf $\mathscr{F}$ on the second countable space $X$ is of type $(\mathscr{N})$. Then $H(U, \mathscr{F})$ is of type $(\mathscr{N})$ for any open subset $U$ of $X$.

Proof. We may assume $U=X$. Let $V$ run through a countable base of open subsets of $X$ such that $H(V, \mathscr{F})$ is of type $(\mathscr{N})$. The restriction maps $H(X, \mathscr{F})$ $\rightarrow H(V, \mathscr{F})$ embed $H(X, \mathscr{F})$ as a closed subspace of the direct product of the $H(V, \mathscr{F})$. Thus, by Lemma 5.1, we need only show:

LEMMA 8.3. Let $E_{i}$ be a sequence of spaces of type $(\mathscr{N})$. Then $F=\prod E_{i}$ is also of type $(\mathscr{N})$.

Proof. We have $F_{c}^{\prime}=\Sigma E_{i c}^{\prime}$ with the inductive limit topology, i.e., the strongest locally convex topology which makes all the maps $E_{i c}^{\prime} \rightarrow \Sigma E_{i c}^{\prime}$ continuous. Let $\left\{\|\cdot\|_{a_{i}}^{i}\right\}$ be a family of Hilbert space (pseudo-) norms defining the topology of $E_{i c}^{\prime}$. The topology of $F_{c}^{\prime}$ is defined by the family of pseudo-norms

$$
\|\| \cdot\|\|_{\alpha, \tau}=\sup _{1 \leqq i \leqq \infty} \tau_{i}\|\cdot\|_{\alpha_{i}}^{i}, \quad \tau=\left\{\tau_{i}\right\}, \alpha=\left\{\alpha_{i}\right\}
$$

where $\tau_{i}$ is a sequence of positive real numbers. The family of Hilbert space norms

$$
\|\cdot\|_{\alpha, \tau}=\left(\Sigma\left(\tau_{i}\|\cdot\|_{\alpha_{i}}^{i}\right)^{2}\right)^{1 / 2}
$$

defines the same topology. Let $H_{\alpha, \tau}$ be the Hilbert space associated to the norm $\|\cdot\|_{\alpha, \tau}$ in $F^{\prime}, H_{\alpha_{i}}^{i}$ the Hilbert space associated to the norm $\|\cdot\|_{\alpha_{i}}$ in $E_{i c}^{\prime}$. Choose $\beta_{i}$ such that $u_{\alpha_{i} \beta_{i}}: H_{\beta_{i}}^{i} \rightarrow H_{\alpha_{i}}^{i}$ has property $(\mathscr{N})$ of $\S 5$,

$$
u_{\alpha_{i} \beta_{i}}=\sum_{j} \lambda_{j}^{i} e_{j}^{i} \otimes f_{j}^{i} \text {. }
$$

We may assume that this map has norm $\leqq 1$, renorming $H_{\beta_{i}}^{i}$ if necessary. Multiplying $\lambda_{i}^{j}, e_{i}^{j}, f_{i}^{j}$ by suitable constants, we may assume that $\left\{\tau_{i} f_{j}^{i}\right\}_{j}$ is bounded by $2^{-i}$ in $H_{\alpha_{i}}^{i}$, and $\Sigma_{j}\left|\lambda_{j}^{i}\right| \leqq 2^{-i}$, and $\left\{e_{j}^{i}\right\}_{j}$ is bounded in $\bar{H}_{\beta_{i}}^{i}$, say by $s_{i}, s_{i}>\tau_{i}$. Then $\left\{e_{j}^{i}\right\}$ is bounded in $\bar{H}_{\beta, s}, \beta=\left\{\beta_{i}\right\}, s=\left\{s_{i}\right\} ;\left\{\tau_{i} f_{j}^{i}\right\}$ is bounded in $H_{\alpha, \tau}$ and $\Sigma_{i j}\left|\lambda_{j}^{i}\right|<\infty$. The map $H_{\beta, s} \rightarrow H_{\alpha, \tau}$ derived from $H_{\beta_{i}}^{i} \rightarrow H_{\alpha_{i}}^{i}$ is given by

$$
\sum_{i j} \lambda^{i} e_{j}^{i} \otimes f_{j}^{i}
$$

9. Values in a locally convex space. Let $E$ be a locally convex space. We associate to each topological sheaf $\mathscr{F}$ on the second countable topological space $X$ a sheaf $\mathscr{F} \varepsilon E$. 
DEFINITION 9.1. $\mathscr{F} \varepsilon E$ is the sheaf on $X$ defined by the presheaf

$$
\{H(U, \mathscr{F}) \varepsilon E, U \subset X \text { open }\} .
$$

In case of a complex manifold $(X, \mathcal{O})$ and a quasi-complete locally convex space $E, \mathcal{O} \& E$ is the sheaf of germs of holomorphic $E$-valued functions; this follows from Corollary 4.2. $\mathcal{O} \varepsilon E$ is in a natural way a topological sheaf. In the general case, $\mathscr{F} \varepsilon E$ is a topological sheaf, too. To prove this we need

Proposition 9.2. Let $\mathscr{F}$ be a topological sheaf on a second countable topological space $X$, and $E$ a locally convex space. We have

$$
H(V, \mathscr{F} \varepsilon E)=H(V, \mathscr{F}) \varepsilon E
$$

for any open set $V \subset X$.

Proof. We may assume $V=X$. Let $r_{U}: H(X, \mathscr{F}) \rightarrow H(U, \mathscr{F}), U \subset X$ open, be the restriction map. The $r_{U}$ induce (continuous) linear maps

$$
H(X, \mathscr{F}) \varepsilon E \rightarrow H(U, \mathscr{F}) \varepsilon E,
$$

hence a linear map

$$
j: H(X, \mathscr{F}) \varepsilon E \rightarrow H(X, \mathscr{F} \varepsilon E) .
$$

This map is injective. For let $f \in \mathscr{L}_{\varepsilon}\left(E_{c}^{\prime}, H(X, \mathscr{F})\right)=H(X, \mathscr{F}) \in E$ with $j(f)=0$. Then there is a basis $\mathscr{U}$ of open sets in $X$ such that $r_{U} \circ f=0$ for $U \in \mathscr{U}$; i.e., for $y \in E^{\prime}, r_{U} \circ f(y)=r_{U}(f(y))=0$ for all $U \in \mathscr{U}$. Hence $f(y)=0$; hence $f=0$.

$j$ is also onto. For let $f \in H(X, \mathscr{F} \varepsilon E)$; there is a basis $\mathscr{U}$ of open sets in $X$ such that for each $U \in \mathscr{U}$ there is $f_{U} \in \mathscr{L}_{\varepsilon}\left(E_{c}^{\prime}, H(U, \mathscr{F})\right)=H(U, \mathscr{F}) \varepsilon E \subset H(U, \mathscr{F} \varepsilon E)$ which coincides with $f \mid U$. Clearly $r_{U W} \circ f_{U}=f_{W}$ for $W \in \mathscr{U}, W \subset U$. Hence the collection $\left\{f_{U}, U \in \mathscr{U}\right\}$ defines an element $g \in \mathscr{L}_{\varepsilon}\left(E_{c}^{\prime}, H(X, \mathscr{F})\right)$. Obviously $j(g)=f$.

COROLlary 9.3. $\mathscr{F} \varepsilon E$ is a topological sheaf in a natural way.

Proof. We give $H(U, \mathscr{F} \varepsilon E)$ the topology of $H(U, \mathscr{F}) \varepsilon E$ if $U$ is an open set. Axiom $\left(\mathscr{F}_{1}\right)$ follows from Proposition 9.2, and axioms $\left(\mathscr{F}_{2}\right)$ and $\left(\mathscr{F}_{3}\right)$ follow immediately from the relation $H(U, \mathscr{F} \varepsilon E)=\mathscr{L}_{\varepsilon}\left(E_{c}^{\prime}, H(U, \mathscr{F})\right)$.

Notice that if $\mathscr{F}$ is a Frechet sheaf and $E$ a Frechet space, then $\mathscr{F} \varepsilon E$ is also a Frechet sheaf, since for an open set $U \subset X, H(U, \mathscr{F}) \varepsilon E$ is a Frechet space as we have seen in $\$ 3$.

Proposition 9.4. Suppose

$$
0 \rightarrow \mathscr{S}_{1} \rightarrow \mathscr{S}_{2} \rightarrow \mathscr{S}_{3} \rightarrow 0
$$

is an exact sequence of coherent analytic sheaves over the analytic space $(X, \mathcal{O})$. Then for any Frechet space $E$, 


$$
0 \rightarrow \mathscr{S}_{1} \varepsilon E \rightarrow \mathscr{S}_{2} \varepsilon E \rightarrow \mathscr{S}_{3} \varepsilon E \rightarrow 0
$$

is exact. If $0 \rightarrow E_{1} \rightarrow E_{2} \rightarrow E_{3} \rightarrow 0$ is an exact sequence of Frechet spaces then

$$
0 \rightarrow \mathscr{F} \varepsilon E_{1} \rightarrow \mathscr{F} \varepsilon E_{2} \rightarrow \mathscr{F} \varepsilon E_{3} \rightarrow 0
$$

is exact for every Frechet sheaf $\mathscr{F}$ on $X$ which is of type $(\mathscr{N})$.

Proof. Each point $x \in X$ has arbitrarily small open neighborhoods $U$ such that

$$
0 \rightarrow H\left(U, \mathscr{S}_{1}\right) \rightarrow H\left(U, \mathscr{S}_{2}\right) \rightarrow H\left(U, \mathscr{S}_{3}\right) \rightarrow 0
$$

is exact; this is a consequence of Theorem $B$ [5; 14]. Thus the first statement follows from Theorem 5.3, and the second one is also immediate by Theorem 5.3.

10. Theorem $\mathbf{A}$ for a sheaf $\mathscr{S} \varepsilon E$. In the following let $(X, \mathcal{O})$ be an analytic space and $E$ a Frechet space. The following lemma establishes the identity of our definition of $E$-valued holomorphic function with a more intuitive one.

Lemma 10.1. Suppose $X$ is a closed subvariety of a polycylinder $P^{n}$ in $\mathbf{C}^{n}$; then every $f \in H\left(X, \mathcal{O}^{E}\right)$ is the restriction to $X$ of a holomorphic E-valued function on $P^{n}$, and conversely.

Proof. Let us denote by $\mathcal{O}_{n}$ the sheaf of germs of holomorphic functions on $P^{n}$. By Theorem B $[5 ; 14]$ we have an exact sequence

$$
H\left(P^{n}, \mathcal{O}_{n}\right) \rightarrow H(X, \mathcal{O}) \rightarrow 0 .
$$

Thus Theorem 5.3 implies that

$$
H\left(P^{n}, \mathcal{O}_{n}\right) \varepsilon E \rightarrow H(X, \mathcal{O}) \varepsilon E \rightarrow 0,
$$

or

$$
H\left(P^{n} ; E\right) \rightarrow H\left(X, \mathcal{O}^{E}\right) \rightarrow 0
$$

is exact, which proves the assertion.

Now we consider again the general case. Let $\mathscr{S}$ be a coherent analytic sheaf on $X$. Note that $\mathscr{S} \& E$ is also an analytic sheaf; the operation of $\mathcal{O}$ on $\mathscr{S} \varepsilon E$ is defined by the continuous maps ( $U \subset X$ open):

$$
\begin{array}{rlrl}
H(U, \mathcal{O}) \times(H(U, \mathscr{S}) \varepsilon E) & \rightarrow H(U, \mathscr{S}) \varepsilon E & =\mathscr{L}_{\varepsilon}\left(E_{c}^{\prime}, H(U, \mathscr{S})\right), \\
f \times g & \rightarrow & f \cdot g & =[f] \circ g
\end{array}
$$

where $[f]$ is the multiplication operator on $H(U, \mathscr{S})$ defined by $f$.

We define now another continuous composition law (continuous in the sense that the map (10.2) is continuous for each open set $U \subset X)$ :

$$
\mathcal{O}^{E} \otimes_{\mathcal{O}} \mathscr{S} \rightarrow \mathscr{S} \varepsilon E
$$

for an open set $U \subset X$ we define the map 


$$
\begin{aligned}
H\left(U, \mathcal{O}^{E}\right) \otimes H(U, \mathscr{S}) & \\
\mathscr{L}_{\varepsilon}\left(E_{c}^{\prime}, H(U, \mathcal{O})\right) \otimes H(U, \mathscr{S}) & \rightarrow H(U, \mathscr{S} \varepsilon E) \\
f \otimes g & \rightarrow f \cdot g=[g] \circ f
\end{aligned}
$$

where the tensor product is taken over the ring $H(U, \mathcal{O})$ and $[g]$ denotes the multiplication map $H(U, \mathcal{O}) \rightarrow H(U, \mathscr{S})$, defined by $g$. We have

Proposition 10.2. $\mathscr{S} \varepsilon E$ is locally finitely generated over $\mathcal{O}^{E}$.

Proof. Let $x \in X$ and $U$ be an open neighborhood of $x$ such that there is an exact sequence

$$
\mathcal{O}^{k} \rightarrow \mathscr{S} \rightarrow 0
$$

of analytic sheaves over $U$. By Proposition 9.4, the sequence

$$
\mathcal{O}^{k} \varepsilon E=\left(\mathcal{O}^{E}\right)^{k} \rightarrow \mathscr{S} \varepsilon E \rightarrow 0
$$

is exact, which just means that the sections $s^{1}, \cdots, s^{k} \in H(U, \mathscr{S})$ defining the sequence (10.3) generate $(\mathscr{S} \varepsilon E)_{y}$ over $\mathcal{O}_{y}^{E}$ at each point $y \in U$.

As a corollary we obtain

TheORem A. Let $(X, \mathcal{O})$ be a Stein analytic space, $\mathscr{S}$ a coherent analytic sheaf over $X$, and $E$ a Frechet space. Then the sections $H(X, \mathscr{S})$ generate the $\operatorname{stalk}\left(\mathscr{S}_{\varepsilon} E\right)_{y}$ over $\mathcal{O}_{y}^{E}$ at each point $y \in X$.

Proof. By Theorem A for $\mathscr{S}[5 ; 14]$, the sections $H(X, \mathscr{S})$ generate $\mathscr{S}_{y}$ over $\mathcal{O}_{y}$ for each $y \in X$. Thus we can find sections $s^{1}, \cdots, s^{k} \in H(X, \mathscr{S})$ defining the exact sequence (10.3). Then (10.4) says that $s^{1}, \cdots, s^{k}$ generate $(\mathscr{S} \varepsilon E)_{y}$ over $\mathcal{O}_{y}^{E}$ at each point $y \in U$.

Let us remark here that, using Proposition 10.2, (10.1) can be shown to be an isomorphism.

Finally let us note the following useful proposition :

Proposition 10.3. Let $Y$ be a closed subvariety of the analytic space $(X, \mathcal{O})$, and $\mathscr{I}(Y)$ resp. $\mathscr{I}(Y, E)$ the subsheaf of $\mathcal{O}$ resp. $\mathcal{O}^{E}$ of germs vanishing on $Y$. Then

$$
\mathscr{I}(Y, E)=\mathscr{I}(Y) \varepsilon E .
$$

Proof. $\mathscr{I}(Y) \varepsilon E$ is a subsheaf of $\mathcal{O}^{E}=\mathcal{O} \varepsilon E$ and clearly contained in $\mathscr{I}(Y, E)$. Let $U \subset X$ be open; $f \in H\left(U, \mathcal{O}^{E}\right)=\mathscr{L}_{\varepsilon}\left(E_{c}^{\prime}, H(U, \mathcal{O})\right)$ belongs to $H(U, \mathscr{I}(Y) \varepsilon E)$ $=\mathscr{L}_{\varepsilon}\left(E_{c}^{\prime}, H(U, \mathscr{I}(Y))\right)$ if and only if $\langle f, y\rangle$ belongs to $H(U, \mathscr{I}(Y))$ for all $y \in E^{\prime}$, i.e., if and only if $f$ vanishes on $Y$.

The last proposition is essential for the applications. Similar propositions hold for other intrinsically defined analytic sheaves; for instance for the sheaf of germs 
in $\mathcal{O}^{E}$ vanishing of second order on $Y$ (meaning $\langle f, y\rangle \in \mathscr{I}(Y)^{2}$ for each $\left.y \in E^{\prime}\right)$, etc. The proofs are identical.

11. Theorems $\mathrm{B}$ and $\mathrm{C}$ for a sheaf $\mathscr{S} \varepsilon E$. Let $\mathscr{S}$ be a coherent analytic sheaf on a Stein space $(X, \mathcal{O})$, and $E$ a Frechet space. Using Theorems $\mathrm{B}$ and $\mathrm{C}$ (Oka-Weil Approximation Theorem) for $\mathscr{S}$ [14, Chapters 3 and 4] we are now in a position to prove similar theorems for $\mathscr{S} \varepsilon E$.

THEOREM B. Let $\mathscr{S}$ be a coherent analytic sheaf on a Stein space $(X, \mathcal{O})$. Then for every Frechet space $E$,

$$
H^{q}(X, \mathscr{S} \varepsilon E)=0 \quad \text { for } q>0 .
$$

Proof. Let $\mathscr{U}$ be a locally finite covering of $X$ by Stein subdomains $U \subset X$ (in the following called a Stein covering of $X)$. The space $C^{q}(\mathscr{U}, \mathscr{S})$ of $q$-cochains on the nerve of $\mathscr{U}$ with coefficients in $\mathscr{S}$ is in a natural way a Frechet space; it is the countable product

$$
C^{q}(\mathscr{U}, \mathscr{S})=\prod H\left(U_{i_{1}}, \cdots, i_{q}, \mathscr{S}\right),
$$

where $U_{i_{1}, \ldots, i_{q}}=U_{i_{1}} \cap \cdots \cap U_{i_{q}}, U_{i_{k}} \in \mathscr{U}$. Moreover, $C^{q}(\mathscr{U}, \mathscr{S})$ is of type $(\mathscr{N})$ by Lemma 8.1 .

Leray's Theorem $\left[17\right.$, Chapter I] says that the cohomology groups $H^{q}(X, \mathscr{S})$ are the cohomology groups of the complex

$$
0 \rightarrow C^{0}(\mathscr{U}, \mathscr{S}) \stackrel{\delta}{\rightarrow} \cdots \rightarrow C^{q}(\mathscr{U}, \mathscr{S}) \rightarrow \cdots
$$

Hence by Theorem B for $\mathscr{S}$,

$$
0 \rightarrow H(X, \mathscr{S}) \stackrel{i}{\rightarrow} C^{0}(\mathscr{U}, \mathscr{S}) \stackrel{\delta}{\rightarrow} \cdots \rightarrow C^{q}(\mathscr{U}, \mathscr{S}) \cdots
$$

is exact. The image of $\delta$ is closed in $C^{q}(\mathscr{U}, \mathscr{S})$ and hence it is of type $(\mathscr{N})$ by Lemma 5.1. Therefore we may apply Theorem 5.3, thus obtaining an exact sequence

$$
0 \rightarrow H(X, \mathscr{S}) \varepsilon E \rightarrow C^{0}(\mathscr{U}, \mathscr{S}) \varepsilon E \rightarrow C^{1}(\mathscr{U}, \mathscr{S}) \varepsilon E \cdots
$$

or

Thus

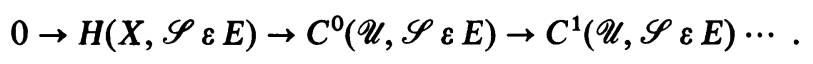

$$
H^{q}(\mathscr{U}, \mathscr{S} \varepsilon E)=Z^{q}(\mathscr{U}, \mathscr{S} \varepsilon E) / B^{q}(\mathscr{U}, \mathscr{S} \varepsilon E)=0
$$

for $q>0$, where

$$
Z^{q}=\delta^{-1}(0) \subset C^{q} \text { and } B^{q}=\delta\left(C^{q-1}\right) \subset C^{q} .
$$

By the definition of Čech cohomology we have

$$
H^{q}(X, \mathscr{S} \varepsilon E)=\text { ind } \lim H^{q}(\mathscr{U}, \mathscr{S} \varepsilon E)=0, \quad q>0,
$$

where $\mathscr{U}$ runs through the set of all locally finite Stein coverings of $X$. 
Leray's Theorem implies now of course $H^{q}(X, \mathscr{S} \varepsilon E)=H^{q}(\mathscr{U}, \mathscr{S} \varepsilon E)$.

Theorem C (OKa-Weil Approximation Theorem). Let $\mathscr{S}$ be a coherent analytic sheaf on a Stein space $(X, \mathcal{O})$, and $E$ any Frechet space. Suppose $U$ is a holomorphically convex subdomain of $X$. The restriction map

$$
H(X, \mathscr{S} \varepsilon E) \rightarrow H(U, \mathscr{S} \varepsilon E)
$$

has a dense image.

Proof. The Oka-Weil Approximation Theorem for $\mathscr{S}$ as proven in [14, Chapter 3] asserts that $H(X, \mathscr{S}) \rightarrow H(U, \mathscr{S})$ has a dense image. Thus $H(X, \mathscr{S}) \otimes E$ has a dense image in $H(U, \mathscr{S}) \otimes E$ (with topology induced by $H(U, \mathscr{S}) \varepsilon E$ ). The latter space is dense in $H(U, \mathscr{S}) \varepsilon E$ since every element of $H(U, \mathscr{S}) \varepsilon E$ is an infinite sum of elements in $H(U, \mathscr{S}) \otimes E$ as we have seen in the proof of Theorem 5.3.

12. Some applications. We are now able to obtain similar consequences as in the scalar case.

Corollary 12.1. Suppose $Y$ is a closed subvariety of a Stein space $(X, \mathcal{O})$. Let $\mathcal{O}^{Y}$ be the sheaf of germs of holomorphic functions on $Y$. Then the restriction map

$$
H\left(X, \mathcal{O}^{E}\right) \rightarrow H\left(Y, \mathcal{O}^{Y} \varepsilon E\right)
$$

is onto for every Frechet space E; i.e., every E-valued holomorphic function on $Y$ is the restriction to $Y$ of an E-valued holomorphic function on $X$.

Proof. The sequence

$$
0 \rightarrow \mathscr{I}(Y, E) \rightarrow \mathcal{O}^{E} \rightarrow \mathcal{O}^{Y} \varepsilon E \rightarrow 0
$$

is exact (by Proposition 9.4 and Proposition 10.4), also if $X$ is not Stein. Now $H^{1}(X, \mathscr{I}(Y, E))=H^{1}(X, \mathscr{I}(Y) \varepsilon E)=0$ and hence the corollary.

Suppose now $E$ is a commutative Frechet algebra (with identity). Let $U$ be an open subset of the analytic space $(X, \mathcal{O})$. We denote by $\mathfrak{M}_{U}^{E}$ the ring of fractions of the ring $H\left(U, \mathcal{O}^{E}\right)$ with respect to the multiplicative system $S$ of nonzero divisors in $H\left(U, \mathcal{O}^{E}\right)$; i.e., $\mathfrak{M}_{U}^{E}$ is the set of pairs $f / g, f \in H\left(U, \mathcal{O}^{E}\right)$ and $g \in S$, where two pairs $f_{1} / g_{1}$ and $f / g$ are identified if $f_{1} \cdot g=f \cdot g_{1} \cdot\left\{\mathfrak{M}_{U}^{E}, U \subset X\right.$ open $\}$ is in a natural way a presheaf. Let $\mathfrak{M}^{E}$ be the corresponding sheaf. $\mathfrak{M}^{E}$ is called the sheaf of germs of $E$-meromorphic functions. $\mathcal{O}^{E}$ is a subsheaf of $\mathfrak{M}^{E}$ by means of the mapping $f_{x} \rightarrow(f / 1)_{x}, x \in X . \mathscr{D}^{E}=\mathfrak{M}^{E} / \mathscr{O}^{E}$ is the sheaf of germs of (additive) $E$-divisors. We have

Proposition 12.2 (Cousin 1). Suppose $(X, \mathcal{O})$ is a Stein space; then

$$
H\left(X, \mathfrak{M}^{E}\right) \rightarrow H\left(X, \mathscr{D}^{E}\right)
$$

is onto; i.e., every E-divisor is defined by an E-meromorphic function.

Proof. By definition 


$$
0 \rightarrow \mathcal{O}^{E} \rightarrow \mathfrak{M}^{E} \rightarrow \mathscr{D}^{E} \rightarrow 0
$$

is exact. Hence by Theorem B

$$
H\left(X, \mathfrak{M}^{E}\right) \rightarrow H\left(X, \mathscr{D}^{E}\right) \rightarrow H^{1}\left(X, \mathcal{O}^{E}\right)=0
$$

is exact.

Let us now point out briefly the relation between these results and some results in the literature. In $[9 ; 6]$ Theorem $A$ is proved for the sheaf of germs of holomorphic cross sections of an analytic vector bundle depending continuously on a parameter $t \in K$, where $K$ is a compact Hausdorff space, i.e., for a sheaf $\mathscr{S} \varepsilon \mathscr{C}(K)$ where $\mathscr{S}$ is a locally free analytic sheaf; that is, a more general theorem is proven (in which the germs are required to be holomorphic only for parameters $t$ in a closed subset $K_{0}$ of $K$ ) from which Theorem A follows (by setting $K_{0}=K$ ).

In [13] a theorem similar to Proposition 12.2 is proven. Let $K$ be a compact Hausdorff space and $\mathcal{O}^{K}$ the sheaf of germs of holomorphic functions on the analytic space $(X, \mathcal{O})$ which depend continuously on $t \in K$, i.e., $\mathcal{O}^{K}=\mathcal{O}^{E}$ where $E=\mathscr{C}(K)$. Define $\mathfrak{M}^{K}$ to be the sheaf of germs of continuous meromorphic families, i.e., $f_{x} \in \mathfrak{M}_{x}^{K}$ has a representation $f=g / h$ with $g, h \in H\left(U, \mathcal{O}^{K}\right)$ in some neighborhood $U$ of $x \in X$ and $h(t)=0$ has codimension $\geqq 1$ at every point for each $t \in K$. (Note that this definition is somewhat different from the definition of $\mathfrak{M}^{E}$ above.) Then $0 \rightarrow \mathcal{O}^{K} \rightarrow \mathfrak{M}^{K} \rightarrow \mathscr{D}^{K}=\mathfrak{M}^{K} / \mathcal{O}^{K} \rightarrow 0$ is exact, and hence $H\left(X, \mathfrak{M}^{K}\right)$ $\rightarrow H\left(X, \mathscr{D}^{K}\right)$ maps onto if $X$ is Stein. This is the theorem proven in [13].

Finally consider a sheaf of ideals $\mathscr{I}$ on the Stein analytic space $(X, \mathcal{O})$ generated by finitely many cross sections $f_{1}, \cdots, f_{k} \in H(X, \mathcal{O})$. Suppose $f_{t} \in H(X, \mathscr{I})$ depends continuously ( $C^{k}$, holomorphically) on a parameter $t \in M$, where $M$ is a locally compact Hausdorff space ( $C^{k}$-manifold, complex manifold); i.e., $f_{t}$ defines an element $g \in H(X, \mathscr{I}) \varepsilon E$ where $E$ is the Frechet space of continuous ( $C^{k}$, holomorphic) functions on $M$ with the topology of uniform convergence (uniform convergence for the first $k$ derivatives) on compact subsets of $M$.(Cf. Proposition 3.1 for the continuous case; the other cases follow similarly.) The $\left(f_{j}\right)$ define an exact sequence

$$
0 \rightarrow \mathscr{K} \rightarrow \mathcal{O}^{k} \rightarrow \mathscr{I} \rightarrow 0
$$

hence

$$
0 \rightarrow \mathscr{K} \varepsilon E \rightarrow\left(\mathcal{O}^{E}\right)^{k}=\mathcal{O}^{k} \varepsilon E \rightarrow \mathscr{I} \varepsilon E \rightarrow 0
$$

is exact, and by Theorem $\mathrm{B}, H\left(X, \mathcal{O}^{E}\right)^{k} \rightarrow H(X, \mathscr{I} \varepsilon E)$ maps onto. Thus we can write

$$
f_{t}=\sum_{1}^{k} g_{t}^{j} \cdot f_{j},
$$


where $g_{t}^{j} \in H(X, \mathcal{O})$ are holomorphic depending continuously $\left(C^{k}\right.$, holomorphic) on $t$. This answers a question raised by Professor Kuranishi.

13. An inverse of the restriction map to a subvariety. In the following let $(X, \mathcal{O})$ be a Stein space and $Y$ a closed subvariety of $X$. We denote the sheaf of germs of holomorphic functions on $Y$ by $\mathcal{O}^{Y}$. We want to construct (in a vague sense) a continuous linear right inverse to the restriction map $H(X, \mathcal{O}) \rightarrow H\left(Y, \mathcal{O}^{Y}\right)$.

We denote by $B(X, \mathcal{O})$ the Banach space of bounded functions in $H(X, \mathcal{O})$. Let $B(X, \mathcal{O})^{*}$ be the dual Banach space of $B(X, \mathcal{O})$. The injection $B(X, \mathcal{O}) \rightarrow H(X, \mathcal{O})$ defines a continuous linear map $H(X, \mathcal{O})_{c}^{\prime} \rightarrow B(X, \mathcal{O})^{*}$ since $H(X, \mathcal{O})_{c}^{\prime}$ has the topology of uniform convergence on bounded sets in $H(X, \mathcal{O})$ (which are compact by Montel's Theorem). Thus, passing to the transpose again, we obtain a continuous linear map

$$
\left[B(X, \mathcal{O})^{*}\right]_{c}^{\prime} \rightarrow H(X, \mathcal{O})
$$

because $\left[H(X, \mathcal{O})_{c}^{\prime}\right]_{c}^{\prime}=H(X, \mathcal{O})[2$, Chapter VI, $\S 3$, Proposition 7 in No. 4 and Theorem 2 in No. 3] $(H(X, \mathcal{O})$ is Montel by Proposition 6.1).

Conversely, every continuous linear map (13.1) determines a continuous linear map $B(X, \mathcal{O}) \rightarrow H(X, \mathcal{O})$ since the canonical injection $B(X, \mathcal{O}) \rightarrow\left[B(X, \mathcal{O})^{*}\right]_{c}^{\prime}$ is continuous.

Now consider the exact sequence

$$
\begin{aligned}
0 \rightarrow \mathscr{I}(Y) \varepsilon B\left(Y, \mathcal{O}^{Y}\right)^{*} & \rightarrow \mathcal{O} \varepsilon B\left(Y, \mathcal{O}^{Y}\right)^{*} \\
& \rightarrow \mathcal{O}^{Y} \varepsilon B\left(Y, \mathcal{O}^{Y}\right)^{*} \rightarrow 0 .
\end{aligned}
$$

By Theorem B,

$$
H(X, \mathcal{O}) \varepsilon B\left(Y, \mathcal{O}^{Y}\right)^{*} \rightarrow H\left(Y, \mathcal{O}^{Y}\right) \varepsilon B\left(Y, \mathcal{O}^{Y}\right)^{*} \rightarrow 0
$$

or

$$
\mathscr{L}\left(\left[B\left(Y, \mathcal{O}^{Y}\right)^{*}\right]_{c}^{\prime}, H(X, \mathcal{O})\right) \rightarrow \mathscr{L}\left(\left[B\left(Y, \mathcal{O}^{Y}\right)^{*}\right]_{c}^{\prime}, H\left(Y, \mathcal{O}^{\mathrm{Y}}\right)\right) \rightarrow 0
$$

is exact. Hence

THeOREM 13.1. Let $(X, \mathcal{O})$ be a Stein space, $Y$ a closed subvariety of $X$, and $B\left(Y, \mathcal{O}^{Y}\right)$ the Banach space of bounded functions in $H\left(Y, \mathcal{O}^{Y}\right)$. The restriction map $H(X, \mathcal{O}) \rightarrow H\left(Y, \mathcal{O}^{Y}\right)$ has a continuous linear right inverse $B\left(Y, \mathcal{O}^{Y}\right) \rightarrow H(X, \mathcal{O})$.

Of course, a similar theorem holds also for coherent analytic subsheaves $\mathscr{S}$ of $\mathcal{O}^{q}$, in which case $B(X, \mathscr{S})$ can be defined. We will later represent this inverse map by an integral formula.

14. Counterexamples. The question arises now whether Theorems $\mathbf{A}$ and $\mathbf{B}$ generalize to the more general situation where $E$ is only required to be quasicomplete; and if not, what can be said at all in the general case. We will see later that Theorem A holds in the general case while Theorem B does not (but Theorem $B$ holds in a weakened form, namely on holomorphically convex compact sets). Here is a counterexample: 
Proposition 14.1. Suppose $(X, \mathcal{O})$ is a connected Stein manifold of dimension $>0$. Let $E$ be the countable direct sum of copies of $\mathbf{C}$ with the topology of the inductive limit (thus $E$ is complete). There is a coherent analytic subsheaf $\mathscr{K}$ of $\mathcal{O}$ with $H^{1}(X, \mathscr{K} \varepsilon E) \neq 0$. (One can in fact prove that $H^{1}(X, \mathscr{K} \varepsilon E) \neq 0$ for any coherent subsheaf $\mathscr{K}$ of $\left.\mathcal{O}^{k}\right)$.

Proof. Let $Y=\left\{a_{n}\right\}$ be a countable closed discrete sequence in $X$. We have an exact sequence

$$
0 \rightarrow \mathscr{K} \rightarrow \mathcal{O} \rightarrow \mathcal{O}^{Y} \rightarrow 0
$$

Obviously, the sequence

$$
0 \rightarrow \mathscr{K} \varepsilon E \rightarrow \mathcal{O}_{\varepsilon} E \rightarrow \mathcal{O}^{Y} \varepsilon E \rightarrow 0
$$

is exact. But $H(X, \mathcal{O} \varepsilon E) \rightarrow H\left(X, \mathcal{O}^{Y} \varepsilon E\right)$ cannot be onto since $H(X, \mathcal{O} \varepsilon E)$ $=\Sigma_{1}^{\infty} H(X, \mathcal{O})$ and $H\left(X, \mathcal{O}^{Y} \varepsilon E\right)=\prod_{1}^{\infty} E$; there is no $E$-valued holomorphic function which has value $e_{n} \in E$ at $a_{n}$, where $e_{n}$ has its first $n$ coordinates equal to 1 and the others equal to 0 . Thus $H^{1}(X, \mathscr{K} \varepsilon E) ! \neq 0$.

There remains still the question whether Theorem 13.1 can be generalized, i.e. whether there exists an inverse $j: H\left(Y, \mathcal{O}^{Y}\right) \rightarrow H(X, \mathcal{O})$. This is certainly false if $X$ is a connected Stein manifold and $Y$ has countably many branches. For then there are $g_{n} \in H\left(Y, \mathcal{O}^{Y}\right), g_{n} \neq 0, n=1, \cdots$, such that $g_{j} \cdot g_{k}=0$ for $j \neq k ; j\left(g_{n}\right)$ vanishes only on a nowhere dense subset of $X$; thus by the Baire category theorem there is a point $x \in X$ such that $j\left(g_{n}(x)\right) \neq 0, n=1, \cdots$. We may assume $j\left(g_{n}(x)\right)=1$; now $g=\Sigma g_{n} \in H\left(Y, \mathcal{O}^{Y}\right)$, but $\Sigma j\left(g_{n}\right)$ does not converge. However, I do not know any counterexample for the case where $Y$ is irreducible, or even a connected submanifold.

15. A generalized Tietze Extension Theorem. In \$18 we want to extend continuous functions with values in the dual of a Banach space from a closed subvariety into the ambient space. We will need the following:

LEMMA 15.1. Let $E$ be a quasi-complete locally convex space which has the property that the compact sets in $E$ are metrisable. Suppose $X$ is a locally compact and paracompact Hausdorff space, and $f$ a continuous $E$-valued function defined on a closed subset $Y$ of $X$. Then there is a continuous E-valued function $f$ on $X$ such that $f^{\prime} \mid Y=f$

Proof. Since we have partitions of unity, we may assume that $X$ is compact and $f$ has values in a compact disc $K$ in $E$. Let $d(\cdot, \cdot)$ be the metric for $K$. We construct by induction on $k$ a sequence $\left(f_{k}\right)_{k=0}^{\infty}$ of continuous $E$-valued function on $X$ such that

(1) $f_{k}(X) \subset K$;

(2) $d\left(\hat{f}_{k}(x)-\hat{f}_{k-1}(x)\right) \leqq 2^{-(k-1)}$ for $x \in X, k>1$;

(3) $d\left(f_{k}(x)-f(x)\right) \leqq 2^{-(k+1)}$ for $x \in Y, k \geqq 1$.

Then $f_{k}$ converges uniformly to a continuous function $f$ on $X$ satisfying $f \mid Y=f$. 
Choose $\hat{f}_{0}=0$, and suppose $f_{k-1}, k>0$, is already defined. Let $\mathscr{U}_{k}=\left\{U_{j}^{k}\right\}$ be a finite covering of $X$ such that

(a) $d(f(x)-f(y)) \leqq 2^{-(k+1)}$ for $x, y \in U_{k}^{j} \cap Y$;

(b) $d\left(\tilde{f}_{k-1}(x)-\tilde{f}_{k-1}(y)\right) \leqq 2^{-k}$ for $x, y \in U_{k}^{j}$.

Choose a partition of unity $\left(e_{k}^{j}\right)$ subordinated to the covering $\mathscr{U}_{k}$. If $U_{k}^{j} \cap Y \neq \varnothing$ pick $x_{k}^{j} \in U_{k}^{j} \cap Y$ and define $c_{k}^{j}=f\left(x_{k}^{j}\right)$. If $U_{k}^{j} \cap Y=\varnothing$ pick $x_{k}^{j} \in U_{k}^{j}$ and define $c_{k}^{j}=\tilde{f}_{k-1}\left(x_{k}^{j}\right)$. Now let

$$
\hat{f_{k}}=\sum_{j} c_{k}^{j} \cdot e_{k}^{j}
$$

$\tilde{f}_{k}$ will have values in $K$ since $K$ is a disc. (2) and (3) are also easily verified.

In the application, $E$ will be the dual of a separable Banach space $B$ considered with the weak topology defined by $B$. It is easily checked that the weak topology for $E$ defined by any dense subset of $B$ induces the same topology in the closed bounded (hence compact) discs that $E$ does. Hence, $B$ being separable, the closed bounded discs in $E$ are metrisable.

16. Digression on holomorphic functions. We give now a characterizaticn of holomorphic functions on an analytic space $(X, \mathcal{O})$ which is similar to the discussion in $\S 4$.

THEOREM 16.1. Let $(X, \mathcal{O})$ be an analytic space and $E$ a quasi-complete locally convex space. Suppose $f$ is an E-valued function on $X$. The following are equivalent:

(1) $f$ is holomorphic, i.e., $f \in \mathscr{L}_{\varepsilon}\left(E_{c}^{\prime}, H(X, \mathcal{O})\right)$;

(2) If $X$ is locally realized as a closed subvariety $V$ of some open neighborhood $U$ of the closure of the unit polycylinder $P^{n}$ in some $\mathbf{C}^{n}$, then there is a holomorphic E-valued function $\tilde{f}$ on $P^{n}$ such that $\tilde{f}\left|V \cap P^{n}=f\right| V \cap P^{n}$;

(3) $f$ is continuous and $\langle f, y\rangle \in H(X, \mathcal{O})$ for each $y \in E^{\prime}$.

Proof. (1) implies (2). We have proved this already for the case that $E$ is a Frechet space. In general, we have to use Theorem 13.1. Let $\eta: B\left(V \cap P^{n}, \mathcal{O}\right)$ $\rightarrow H\left(P^{n}, \mathcal{O}_{n}\right)\left(\mathcal{O}_{n}\right.$ the sheaf of germs of holomorphic functions on $\left.P^{n}\right)$ be a continuous linear right inverse to the restriction map $H\left(P^{n}, \mathcal{O}_{n}\right) \rightarrow H\left(V \cap P^{n}, \mathcal{O}\right)$.

$$
f \mid\left(V \cap P^{n}\right) \in \mathscr{L}_{\varepsilon}\left(E_{c}^{\prime}, B\left(V \cap P^{n}, \mathcal{O}\right)\right)
$$

thus $\eta \circ\left(f \mid V \cap P^{n}\right)=\hat{f} \in \mathscr{L}_{\varepsilon}\left(E_{c}^{\prime}, H\left(P^{n}, \mathcal{O}_{n}\right)\right) . \tilde{f}$ is holomorphic by Theorem 4.1 and clearly $\hat{f}\left|V \cap P^{n}=f\right| V \cap P^{n}$.

(2.) implies (3) is trivial.

(3) implies (1). $f \in \mathscr{L}_{\varepsilon}\left(E_{c}^{\prime}, H(X, \mathscr{C})\right)$ by Proposition 3.1 , and by assumption, $f$ is in the subspace $\mathscr{L}_{\varepsilon}\left(E_{c}^{\prime}, H(X, \mathcal{O})\right)$ of $\mathscr{L}_{\varepsilon}\left(E_{c}^{\prime}, H(X, \mathscr{C})\right)$.

We will see in $\S 19$ that we need not require $f$ to be continuous in (3) of Theorem 16.1 .

THEOREM 16.2.: Let $(X, \mathcal{O})$ be an analytic space and $E$ a vector space. Suppose 
$\alpha$ and $\beta$ are two quasi-complete locally convex topologies for $E$ which have the same bounded sets. An E-valued function on $X$ is holomorphic for $\alpha$ if and only if it is holomorphic for $\beta$.

Proof. Let $E_{\alpha}$ resp. $E_{\beta}$ be the space $E$ endowed with the topology $\alpha$ resp. $\beta$. Suppose $f$ is an $E_{\alpha}$-valued holomorphic function. In view of (2) in Theorem 16.1, we may assume that $X$ is a polycylinder $P^{n}$ in some $\mathbf{C}^{n}$, and that $f$ has a power series expansion (Theorem 4.1)

$$
f(z)=\sum a_{\sigma} z^{\sigma}
$$

where $\left\{a_{\sigma}\right\}$ is a bounded set in $E_{\alpha}$. Hence $\left\{a_{\sigma}\right\}$ is bounded in $E_{\beta}$ and the power series on the right of (16.1) converges to a holomorphic $E_{\beta}$-valued function $g$ on $P^{n}$. It remains to be shown that $f=g$. Let $\gamma$ be the supremum of the topologies $\alpha$ and $\beta$. The bounded sets in $E_{\gamma}$ are the same as in $E_{\alpha}$ and $E_{\beta}$. Thus the power series on the right of (16.1) converges to an $E_{\gamma}$-valued function $h$ on $P^{n}$. Since the injections $E_{\gamma} \rightarrow E_{\alpha}$ and $E_{\gamma} \rightarrow E_{\beta}$ are continuous we conclude $f(z)=h(z)=g(z)$ for $z \in P^{n}$.

17. Some generalizations. Suppose $E$ is a quasi-complete locally convex space and $B$ a closed bounded disc in $E$. We denote by $E_{B}$ the subspace of $E$ generated by $B . E_{B}$ is considered with the topology defined by the norm whose closed unit ball is $B$. It is known that since $B$ is complete in $E, B$ is also complete in $E_{B}$ and thus $E_{B}$ is a Banach space [2, Chapter I, $\S 1$, Proposition 8 in No. 5]. If $B \subset A$, then $E_{B} \rightarrow E_{A} \rightarrow E$ are continuous.

Now consider an analytic space $(X, \mathcal{O})$ and suppose $\mathscr{S}$ is a coherent analytic sheaf on $X$. We know that if $V \subset U$ are open sets and $V$ is relatively compact in $U$, then $r: H(U, \mathscr{S}) \rightarrow H(V, \mathscr{S})$ is compact. This implies that the transpose $r^{\prime}: H(V, \mathscr{S})_{c}^{\prime} \rightarrow H(U, \mathscr{S})_{c}^{\prime}$ is compact, too; for if $W$ is a neighborhood of 0 in $H(U, \mathscr{S})$ such that $r(W)$ is contained in a compact disc $K$, then the neighborhood $K^{0}$ of 0 in $H(V, \mathscr{S})_{c}^{\prime}$ is mapped into $W^{0}$ which is compact. Now we can prove:

THEOREM 17.1. Let $(X, \mathcal{O})$ be an analytic space, and $\mathscr{S}$ a coherent analytic sheaf on $X$. For any quasi-complete locally convex space $E$

$$
\mathscr{S} \varepsilon E=\text { ind } \lim \mathscr{S} \varepsilon E_{B} \text { (algebraically!) }
$$

where $B$ runs through the set of closed bounded discs in $E$.

Proof. The continuous maps $E_{B} \rightarrow E$ define a map ind $\lim \mathscr{S} \varepsilon E_{B} \rightarrow \mathscr{S} \varepsilon E$ which is clearly injective. Now let $f_{x} \in(\mathscr{S} \varepsilon E)_{x}$, say $f_{x}$ is defined by $f \in H(U, \mathscr{S} \varepsilon E)$ for some open neighborhood $U$ of $x$. Let $V \subset U$ be an open neighborhood of $x$ which is relatively compact in $U$. We have $f \in \mathscr{L}\left(H(U, \mathscr{S})_{c}^{\prime}, E\right)$. Let $W$ be a neighborhood of 0 in $H(V, \mathscr{S})_{c}^{\prime}$ such that $r^{\prime}(W)$ is relatively compact in $H(U, \mathscr{S})_{c}^{\prime}$ $(r: H(U, \mathscr{S}) \rightarrow H(V, \mathscr{S})$ is the restriction map). Now $f$ considered as an element of $\mathscr{L}\left(H(V, \mathscr{S})_{c}^{\prime}, E\right)$ maps $W$ into a closed bounded disc $B \subset E$, i.e., $f$ is in the image of the injection $\mathscr{L}_{\varepsilon}\left(H(V, \mathscr{S})_{c}^{\prime}, E_{B}\right) \rightarrow \mathscr{L}_{\varepsilon}\left(H(V, \mathscr{S})_{c}^{\prime}, E\right)$. 
Corollary 17.2. Suppose

$$
0 \rightarrow \mathscr{S}_{1} \rightarrow \mathscr{S}_{2} \rightarrow \mathscr{S}_{3} \rightarrow 0
$$

is an exact sequence of coherent analytic sheaves on the analytic space $(X, \mathcal{O})$. For any quasi-complete locally convex space $E$,

$$
0 \rightarrow \mathscr{S}_{1} \varepsilon E \rightarrow \mathscr{S}_{2} \varepsilon E \rightarrow \mathscr{S}_{3} \varepsilon E \rightarrow 0
$$

is also exact.

Proof. We have only to apply Proposition 9.4 and Theorem 17.1

We define the the continuous composition law

$$
\mathcal{O}^{E} \otimes_{\mathcal{O}} \mathscr{S} \rightarrow \mathscr{S} \varepsilon E
$$

for any quasi-complete locally convex space as in $\$ 10$. The proofs of Proposition 10.2 and Theorem $\mathrm{A}$ transfer now to the general case and yield:

COROLlaRY 17.3. Let $\mathscr{S}$ be a coherent analytic sheaf on the analytic space $(X, \mathcal{O})$, and $E$ a quasi-complete locally convex space. $\mathscr{S} \varepsilon E$ is locally finitely generated over $\mathcal{O}^{E}$.

THEOREM A. Suppose moreover that $(X, \mathcal{O})$ is Stein. Then the cross sections $H(X, \mathscr{S})$ generate the stalk $(\mathscr{S} \varepsilon E)_{x}$ over $\mathcal{O}_{*}^{E}$ at each point $x \in X$.

Finally let $K$ be a holomorphically convex compact subset of the Stein space $(X, \mathcal{O})$, and $\mathscr{S}$ a coherent analytic sheaf on $X$. Recall that for any sheaf $\mathscr{F}$ of vector spaces on $X$

$$
H^{q}(K, \mathscr{F})=\text { ind } \lim H^{q}(U, \mathscr{F}) \quad[7, \text { Chapter II ; 4], }
$$

where $U$ runs through the set of open neighborhoods of $K$. Hence $H^{q}(K, \mathscr{S} \varepsilon E)=0$ for $q>0$ and every Frechet space $E$ (Theorem B). On the other hand, if the sheaf $\mathscr{F}$ is the inductive limit of a system $\left\{\mathscr{F}_{\alpha}\right\}$ of sheaves of vector spaces, then

$$
H^{q}(K, \mathscr{F})=\text { ind } \lim H^{q}\left(K, \mathscr{F}_{\alpha}\right) \quad[7, \text { Chapter II; 4]. }
$$

Thus Theorem 17.1 implies

TheOREM B*. Let $\mathscr{S}$ be a coherent analytic sheaf on the Stein space $(X, \mathcal{O})$ and $K$ a holomorphically convex compact subset of $X$. Then

$$
H^{q}(K, \mathscr{S} \varepsilon E)=0, \quad q>0,
$$

for any quasi-complete locally convex space $E$.

18. An integral representation of the inverse of the restriction map to a subvariety. Let $(X, \mathcal{O})$ be a Stein analytic space and $Y$ a closed subvariety of $X$ and $\mathcal{O}^{Y}$ the 
sheaf of germs of holomorphic functions on $Y$. In $\$ 13$ we have constructed a continuous linear right inverse $j: B\left(Y, \mathcal{O}^{Y}\right) \rightarrow H(X, \mathcal{O})$ to the restriction map $H(X, \mathcal{O}) \rightarrow H\left(Y, \mathcal{O}^{Y}\right)$. We are now going to represent a choice of this map $j$ by an integral formula.

Let $\mathfrak{M}$ be the space of bounded measures on $Y$, i.e., $\mathfrak{M}$ is the dual of $B(Y, \mathscr{C})$, the Banach space of bounded continuous functions on $Y$. We consider $\mathfrak{M}$ with the weak topology defined by $B(Y, \mathscr{C})$, while we denote the dual Banach space of $B(Y, \mathscr{C})$ by $B(Y, \mathscr{C})^{*} \cdot B\left(Y, \mathcal{O}^{Y}\right)$ is a closed subspace of $B(Y, \mathscr{C})$. Let $\mathscr{A} \subset \mathfrak{M}$ be the space of measures which annihilate $B\left(Y, \mathcal{O}^{Y}\right)$.

Consider now the function

$$
\phi: Y \rightarrow \mathfrak{M}, \phi(x)=\delta_{x},
$$

where $\delta_{x}$ is the unit point mass at $x \in Y$. Let $\pi: \mathfrak{M} \rightarrow \mathfrak{M} / \mathscr{A}$ be the quotient map and define $\bar{\phi}=\pi \circ \phi ; \phi$ is continuous. The dual of $\mathfrak{M} \mid \mathscr{A}$ is $B\left(Y, \mathcal{O}^{\mathrm{Y}}\right)$, and $\langle\bar{\phi}, f\rangle=f$ is holomorphic for $f \in B\left(Y, \mathcal{O}^{Y}\right)$. Thus by Theorem $16.1, \bar{\phi}$ is a holomorphic $(\mathfrak{M} / \mathscr{A})$-valued function on $Y$. Since $\mathfrak{M} / \mathscr{A}$ and $B\left(Y, \mathcal{O}^{Y}\right)^{*}$ are quasi-complete and have the same bounded sets, we may apply Theorem 16.2 , thus $\phi$ is a holomorphic $B\left(Y, \mathcal{O}^{Y}\right)^{*}$-valued function on $Y$. By Corollary $12.1, \Phi$ is the restriction to $Y$ of a holomorphic $B\left(Y, \mathcal{O}^{Y}\right)^{*}$-valued function $\tau$ on $X$. Now

$$
0 \rightarrow \mathcal{O} \varepsilon\left[B(Y, \mathscr{C}) / B\left(Y, \mathcal{O}^{Y}\right)\right]^{*} \rightarrow \mathcal{O} \varepsilon B(Y, \mathscr{C})^{*} \rightarrow \mathcal{O} \varepsilon B\left(Y, \mathcal{O}^{Y}\right)^{*} \rightarrow 0
$$

is exact by Proposition 9.4. Hence by Theorem $B$,

$$
H\left(X, \mathcal{O} \& B(Y, \mathscr{C})^{*}\right) \rightarrow H\left(X, \mathcal{O} \varepsilon B\left(Y, \mathcal{O}^{Y}\right)^{*}\right)
$$

maps onto. So we can find a holomorphic $\mathfrak{M}$-valued function $\eta$ such that $\pi \circ \eta=\tau$. $\eta$ defines a continuous mapping

$$
\begin{aligned}
B\left(Y, \mathcal{O}^{Y}\right) & \rightarrow H(X, \mathcal{O}), \\
f & \rightarrow f, \quad f(z)=\int_{Y} f d \eta_{z},
\end{aligned}
$$

where $\eta_{z}$ denotes the value of $\eta$ at $z \in X$. We have $f \mid Y=f$ by construction.

However $\eta \mid Y \neq \phi$; in fact $\phi$ is not even continuous if considered as a function with values in $B(Y, \mathscr{C})^{*}$ (unless $Y$ is discrete). Nevertheless, we can construct a continuous $\mathfrak{M}$-valued function $\eta^{0}$ on $X$ such that $\eta^{0} \mid Y=\phi$ and the integral formula in (18.1) holds if we replace $\eta$ by $\eta^{0}$. For let $\phi^{0}=(\eta \mid Y)-\phi$; then $\phi^{0}$ has values in $\mathscr{A}$ and is continuous. By Theorem 15.1, $\phi^{0}=\tau^{0} \mid Y$ for a continuous $\mathscr{A}$-valued function $\tau^{0}$ on $X$. Now define

$$
\eta^{0}=\eta-\tau^{0}
$$

$\eta^{0}$ has the desired property. Thus

TheOREM 18.1. Let $(X, \mathcal{O})$ be a Stein space and $Y$ a closed subvariety of $X$. Let $\mathfrak{M}$ be the space of bounded measures on $Y$ considered with the weak topology. 
(1) There is a holomorphic $\mathfrak{M}$-valued function $\eta$ on $X$ such that

$$
\tilde{f}(z)=\int_{Y} f d \eta_{z}
$$

defines a holomorphic extension of $f \in B\left(Y, \mathcal{O}^{Y}\right)$ to all of $X$.

(2) There is a continuous $\mathfrak{M}$-valued function $\eta^{0}$ on $X$ such that $\eta_{y}^{0}=\delta_{y}$ (unit point mass at $y$ ) for $y \in Y$ and

$$
\hat{f}(z)=\int_{Y} f d \eta_{z}^{0}
$$

defines a holomorphic extension of $f \in B\left(Y, \mathcal{O}^{Y}\right)$ to all of $X$.

These integral formulas hold of course also for holomorphic functions with values in a quasi-complete locally convex space $E$ :

Corollary 18.2. If $f$ is a bounded E-valued holomorphic function on $Y$ (meaning $f(Y)$ is bounded in $E$ ), then

$$
\hat{f}(z)=\int_{Y} f d \eta_{z} \quad\left(\operatorname{resp} \cdot \hat{f}(z)=\int_{Y} f d \eta_{z}^{0}\right)
$$

defines a holomorphic extension of $f$ to all of $X$.

Proof. $\langle\tilde{f}, y\rangle=\int_{Y}\langle f, y\rangle d \eta$ is holomorphic for $y \in E^{\prime}$ by Theorem 18.1. Since $\hat{f}=\int_{Y} f d \eta$ is clearly continuous ( $\eta$ being continuous for the strong topology in $\mathfrak{M}$ ), we may apply Theorem 16.1 and conclude that $\tilde{f}$ is holomorphic. In the case of $\eta^{0}$ it is not obvious that $\tilde{f}=\int_{Y} f d \eta^{0}$ is continuous; however we have announced already the fact that the continuity requirement in (3) of Theorem 16.1 is unnecessary. $\tilde{f} \mid Y=f$ is obviously fulfilled.

19. Cauchy integral formulas: general case. Let $U$ be a relatively compact subdomain of an analytic space $(X, \mathcal{O})$. We denote by $A(\bar{U}, \mathcal{O})$ the closure of $H(\bar{U}, \mathcal{O})$ in $H(\bar{U}, \mathscr{C})$ (the Banach space of continuous functions on $\bar{U})$. Let $\Gamma$ be the Šilov boundary of the algebra $A(\bar{U}, \mathcal{O}) ; \Gamma$ is the smallest closed subset of $\bar{U}$ on which each function $f \in A(\bar{U}, \mathcal{O})$ attains its maximum, and which has the property that $y \in \Gamma$ whenever there is $x \in \Gamma$ such that $f(x)=f(y)$ for all $f \in A(\bar{U}, \mathcal{O})$. $A(\bar{U}, \mathcal{O})$ is a closed subspace of $H(\Gamma, \mathscr{C})$. Let $\mathfrak{M}$ be the vector space of measures on $\Gamma$ endowed with the weak topology. We have

THEOREM 19.1. There is a holomorphic M-valued function $\eta$ on $U$ such that

$$
f(z)=\int_{\Gamma} f d \eta_{z} \quad\left(\eta_{z} \text { value of } \eta \text { at } z \in U\right) \text { for } f \in A(\bar{U}, \mathcal{O}) \text {. }
$$

REMARK (ADDED IN PROOF). In his paper, The abstract theorem of Cauchy-Weil (Pacific J. Math. 12 (1962), 511-525), A. Gleason proves a somewhat stronger 
theorem for complex manifolds. But his Theorem 4.8 is also valid for analytic spaces as we have seen in Theorem 5.3; thus the proof of Gleason's Theorem transfers word for word to the case of an analytic space.

Proof. We let $\mathscr{A}$ be the subspace of $\mathfrak{M}$ of measures which annihilate $A(\bar{U}, \mathcal{O})$. $\mathfrak{M} / \mathscr{A}$ is the dual of $A(\bar{U}, \mathcal{O})$ with the weak topology defined by $A(\bar{U}, \mathcal{O})$. We define the continuous function

$$
\phi: U \rightarrow \mathfrak{M} / \mathscr{A}, \quad \phi(x)=\delta_{x},
$$

where $\delta_{x}$ is the evaluation at $x \in U$. Since $A(\bar{U}, \mathscr{O})$ is the dual of $\mathfrak{M} / \mathscr{A}$ and $\langle\phi, f\rangle=f \mid U$ is holomorphic on $U$ for $f \in A(\bar{U}, \mathcal{O})$, Theorem 16.1 implies that $\phi$ is a holomorphic $(\mathfrak{M} / \mathscr{A})$-valued function. Theorem 16.2 assures us that $\phi$ is in fact a holomorphic $A(\bar{U}, \mathcal{O})^{*}$-valued function. By Theorem 5.3

$$
H\left(U, \mathcal{O} \& H(\Gamma, \mathscr{C})^{*}\right) \rightarrow H\left(U, \mathcal{O} \varepsilon A(\bar{U}, \mathcal{O})^{*}\right)
$$

maps onto, which means that we can find a holomorphic $\mathfrak{M}$-valued function $\eta$ such that $\pi \circ \eta=\phi(\pi: \mathfrak{M} \rightarrow \mathfrak{M} / \mathscr{A}$ the quotient map). Clearly $\eta$ has the desired property.

As in $\S 18$ we have the

COROLlaRY 19.2. Let $E$ be a quasi-complete locally convex space. If $f$ is an E-valued holomorphic function defined and holomorphic in a neighborhood of $\bar{U}$, then

$$
f(z)=\int_{\Gamma} f d \eta_{z}, \quad z \in U .
$$

Now let us prove that the continuity requirement in (3) of Theorem 16.1 can be omitted:

COROLLARY 19.3. Suppose $E$ is a locally convex space and $f$ an E-valued function on the analytic space $(X, \mathcal{O})$ such that $\langle f, y\rangle \in H(X, \mathcal{O})$ for $y \in E^{\prime}$. Then $f$ is continuous (and hence holomorphic if $E$ is quasi-complete).

Proof. The proof is similar to the proof of Theorem I in [11]. Let $x \in X$ and $U$ be an open relatively compact set containing $x . f$ is a continuous $E_{s}$-valued function. Let $K$ be the closed convex hull of $f(\Gamma)$ in the completion $\hat{E}_{s}$ of $E_{s}\left(\hat{E}_{s}\right.$ is the algebraic dual of $\left.E^{\prime}\right)$. For any $z \in U$ we have

$$
f(z)-f(x)=\int_{\Gamma} f d\left(\eta_{z}-\eta_{x}\right) \in\left\|\eta_{z}-\eta_{x}\right\| \cdot K .
$$

In fact, $f(z)-f(x) \in\left\|\eta_{z}-\eta_{x}\right\| \cdot(K \cap E)$. The assertion follows now from the fact that $K \cap E$ is bounded and $\eta_{z}$ is strongly continuous in $z$ (because it is holomorphic). 
Finally let us note that we can combine Theorem 18.1 and Theorem 19.1:

Corollary 19.4. Suppose $(X, \mathcal{O})$ is a closed subvariety of a Stein space $\left(M, \mathcal{O}^{M}\right)$. Suppose $W$ is a holomorphically convex subdomain of $M$ such that $U=X \cap W$ is relatively compact and nonempty. There is a holomorphic $\mathfrak{M}$-valued function $\eta$ on $W$ such that for $f \in A(\bar{U}, \mathcal{O})$

$$
f(z)=\int_{\Gamma} f d \eta_{z}, \quad z \in W,
$$

defines a holomorphic extension of $f$ into $W$.

Proof. Let $\eta^{1}$ be the $\mathfrak{M}$-valued holomorphic function on $U$ of Theorem 19.1, and $\eta^{2}$ the holomorphic function on $W$ with values in the space of bounded measures on $U$ constructed in Theorem 18.1. Then

will do it.

$$
\eta_{z}=\int_{U} \eta^{1} d \eta_{z}^{2}
$$

20. Cauchy integral formulas for domains in $\mathbf{C}^{n}$. We obtain a result somewhat stronger than Theorem 19.1 if we restrict our attention to relatively compact subdomains $D$ in $\mathbf{C}^{n}$ with smooth boundaries; we have, however, to use an integral formula discovered by E. Martinelli [12] and S. Bochner [1]. Before going into detail let us remark:

LEMMA 20.1. Let $E$ be a quasi-complete locally convex space and $f$ a continuous E-valued function on the analytic space $(X, \mathcal{O})$. Suppose $\langle f, y\rangle \in H(X, \mathcal{O})$ for $y$ in a dense subspace of $E_{j}^{\prime}$. Then $f$ is holomorphic.

Proof. $f \in \mathscr{L}\left(E_{c}^{\prime}, H(X, \mathscr{C})\right)$ by Proposition 3.1 , where $\mathscr{C}$ is the sheaf of germs of continuous functions on $X$. In particular, $f$ defines a continuous linear map $E_{c}^{\prime} \rightarrow H(X, \mathscr{C})$. By assumption, this map has its image in the closed subspace $H(X, \mathcal{O})$ of $H(X, \mathscr{C})$, i.e., $f \in \mathscr{L}\left(E_{c}^{\prime}, H(X, \mathcal{O})\right)$ and $f$ is holomorphic.

Now let us recall E. Martinelli's integral formula. In $\mathbf{C}^{n}$ let

$$
\beta_{z}=\frac{(n-1) !}{(2 \pi i)^{n}} \cdot \frac{1}{|w-z|^{2^{n}}} \sum_{1}^{n}\left(\bar{w}_{j}-\bar{z}_{j}\right) \sigma^{j}
$$

where

$$
\sigma^{j}=d w_{1} \wedge d \bar{w}_{1} \wedge \cdots \wedge d w_{j} \wedge\left[d \bar{w}_{j}\right] \wedge \cdots \wedge d \bar{w}_{n}
$$

$\left(d \bar{w}_{j}\right.$ being omitted). If $D$ is a relatively compact connected subdomain of $\mathbf{C}^{n}$ with piecewise smooth boundary $\partial D$, and $f$ is holomorphic in a neighborhood of $\bar{D}$, then

$$
f(z)=\int_{\partial D} f \beta_{z} \quad \text { for } z \in D
$$


By piecewise smooth boundary we mean that $\partial D$ is a $(2 n-1)$-dimensional manifold which possesses a triangulation $\partial D=\bigcup_{1}^{n} \Delta_{k}$ such that each $\Delta_{k}$ is the image of the standard (2n-1)-simplex $\Delta$ in $\mathbf{R}^{2 n}$ under a map of class $C^{2}$ which gives a nonsingular imbedding of a neighborhood of $\Delta$ into $\mathbf{C}^{n}$.

Suppose now $\partial D$ is a $C^{\infty}$ manifold and let $\Omega$ be the Frechet space of $C^{\infty}(2 n-1)$ forms on $\partial D . \Omega$ is a Montel space, thus $\Omega$ is the dual of $\Omega_{c}^{\prime}$ [16a, Chapter III]. We have a natural continuous injection

$$
j: A(\bar{D}, \mathcal{O}) \rightarrow \Omega_{c}^{\prime}
$$

mapping $f \in A(\bar{D}, \mathcal{O})$ into $\left(\alpha \rightarrow \int f \alpha\right)$. Let $\mathscr{A} \subset \Omega$ be the annihilator of $j(A(\bar{D}, \mathcal{O}))$ and $\pi: \Omega \rightarrow \Omega / \mathscr{A}$ the quotient map. By Martinelli's Theorem there is a continuous map

such that

$$
\beta: D \rightarrow \Omega
$$

$$
f(z)=\int_{\partial D} f \beta_{z} \quad \text { for } f \in A(\bar{D}, \mathcal{O}), z \in D .
$$

Since $\overline{j(A(\bar{D}, \mathcal{O}))}$ is the dual of $\Omega / \mathscr{A}$ we conclude by Lemma 20.1 that $\pi \circ \beta$ is a holomorphic $(\Omega / \mathscr{A})$-valued function on $D$. As in the proof of Theorem 19.1 we can lift $\pi \circ \beta$ to a holomorphic function $\alpha: D \rightarrow \Omega$. Thus

THEOREM 20.2. Let $D$ be a relatively compact connected subdomain of $\mathbf{C}^{n}$ with $C^{\infty}$ boundary $\partial D$, and $\Omega$ the Frechet space of $C^{\infty}(2 n-1)$-forms on $\partial D$. There is a holomorphic map $\alpha: D \rightarrow \Omega$ such that

$$
f(z)=\int_{\partial D} f \alpha_{z} \quad \text { for } f \in A(\bar{D}, \mathcal{O}), z \in D .
$$

Unfortunately, these abstract existence theorems do not show how to obtain an explicit formula in the applications. Moreover, one would like to know whether $\alpha$ can be chosen to be a kernel. We will explore this question in a forthcoming paper based on the last portion of the author's thesis.

\section{BIBLIOGRAPHY}

1. S. Bochner, Analytic and meromorphic continuation by means of Green's formula, Ann. of Math. (2) 44 (1943), 652-673.

2. N. Bourbaki, Espaces vectoriels topologiques, Hermann, Paris, 1953/55.

3. - Integration, Hermann, Paris, 1960.

4. L. Bungart and H. Rossi, On the closure of certain spaces of holomorphic functions, Math. Ann. (to appear).

5. H. Cartan, Séminaire, École Normale Supérieure, 1951/52.

6. - Espaces fibrés analytiques (à la H. Grauert), Symposium International de Topologia Algebraica (1958), Universidad National Autónoma de Mexico and UNESCO.

7. R. Godement, Topologie algébrique et théorie des faisceaux, Hermann, Paris, 1958.

8. H. Grauert and R. Remmert, Komplexe Räume, Math. Ann. 136 (1958), 245-318. 
9. H. Grauert, Approximationssätze für holomorphe Funktionen mit Werten in komplexen Räumen, Math. Ann. 133 (1957), 139-159.

9a. - Holomorphe Funktionen mit Werten in komplexen Lieschen Gruppen, Math. Ann. 133 (1957), 450-472.

10. A. Grothendieck, Produit tensoriels topologiques et espaces nucléaires, Mem. Amer. Math. Soc. No. 16 (1955).

11. - Sur certains espaces de fonctions holomorphes. I, J. Reine Angew. Math. 192 (1953), 35-64.

12. E. Martinelli, Alcuni teoremi integrali per le funzioni analytiche di più variabili complesse, Rend. Accad. Italia 9 (1938), 269.

13. R. Narasimhan, The Levi problem for complex spaces, Math. Ann. 142 (1961), 355-365.

14. H. Rossi, Global theory of several complex variables, Lecture notes by $L$. Bungart, Princeton Univ., Princeton, N. J., Spring 1961.

15. L. Schwartz, Produit tensoriels topologiques et espaces nucléaires, Séminaire Inst. Henri Poincaré, 1953/54.

16. - Théorie des distributions à valeurs vectorieles. I, Ann. Inst. Fourier (Grenoble) 7 (1957), 1-141.

16a. - Théorie des distributions. I, Hermann, Paris, 1959.

17. J.-P. Serre, Faisceaux algébriques cohérents, Ann of Math. (2) 61 (1955), 197-278.

Princeton UNIVERSITY,

Princeton, New Jersey 